DR. PRATISHTHA CHATTERJEE (Orcid ID : 0000-0003-4877-1958)

DR. STEVE PEDRINI (Orcid ID : 0000-0002-6409-8022)

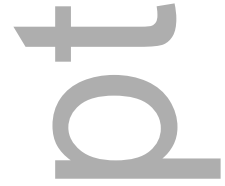

Article type : Original Article

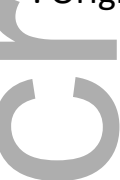

\title{
Plasma metabolites associated with biomarker evidence of neurodegeneration in cognitively normal older adults
}

Pratishtha Chatterjee ${ }^{1,2, *}$, Yeo-Jin Cheong ${ }^{1}$, Atul Bhatnagar ${ }^{6}$, Kathryn Goozee ${ }^{1,3,4,5}$, Yunqi $\mathrm{Wu}^{6}$, Matthew McKay ${ }^{6}$, Ian J. Martins ${ }^{2}$, Wei L.F. Lim ${ }^{2}$, Steve Pedrini' ${ }^{2}$, Michelle Tegg 2 , Victor L. Villemagne ${ }^{7}$, Prita R. Asih ${ }^{2}$, Preeti Dave ${ }^{1,4}$, Tejal M. Shah ${ }^{1,2,8}$, Cintia B. Dias ${ }^{1}$, Stephanie J. Fuller $^{1}$, Heidi Hillebrandt ${ }^{1}$, Sunil Gupta ${ }^{1}$, Eugene Hone ${ }^{2}$, Kevin Taddei ${ }^{2,8}$, Henrik Zetterberg ${ }^{9}$, 10,11,12, Kaj Blennow ${ }^{9,10}$, Hamid R. Sohrabi ${ }^{1,2,8,13}$, Ralph N. Martins ${ }^{1,2,3,5,8, *}$

${ }^{1}$ Department of Biomedical Sciences, Macquarie University, North Ryde, NSW, Australia

${ }^{2}$ School of Medical and Health Sciences, Edith Cowan University, Ralph \& Patricia Sarich Neuroscience Research Institute, Nedlands, WA, Australia

${ }^{3}$ KaRa Institute of Neurological Disease, Sydney, Macquarie Park, NSW, Australia

${ }^{4}$ Clinical Research Department, Anglicare, Sydney, Castle Hill, NSW, Australia

${ }^{5}$ School of Psychiatry and Clinical Neurosciences, University of Western Australia, Crawley, WA, Australia

${ }^{6}$ Department of Molecular Sciences, Macquarie University, North Ryde, NSW, Australia

${ }^{7}$ The Florey Institute of Neuroscience and Mental Health, University of Melbourne, VA, Australia

This is the author manuscript accepted for publication and has undergone full peer review but has not been through the copyediting, typesetting, pagination and proofreading process, which may lead to differences between this version and the Version of Record. Please cite this article as doi: $10.1111 / \mathrm{JNC} .15128$

This article is protected by copyright. All rights reserved 
${ }^{8}$ Australian Alzheimer's Research Foundation, Nedlands, WA, Australia

${ }^{9}$ Department of Psychiatry and Neurochemistry, Institute of Neuroscience and Physiology,

University of Gothenburg, Mölndal, Sweden

${ }^{10}$ Clinical Neurochemistry Laboratory, Sahlgrenska University Hospital, Mölndal, Sweden

11 Department of Neurodegenerative Disease, UCL Institute of Neurology, Queen Square,

London, United Kingdom

${ }^{12}$ UK Dementia Research Institute at UCL, London, United Kingdom

${ }^{13}$ Centre for Healthy Ageing, School of Psychology and Exercise Science, College of Science, Health, Engineering and Education, Murdoch University, Murdoch, WA, Australia

\section{*Corresponding authors:}

Dr Pratishtha Chatterjee, Department of Biomedical Sciences, Macquarie University, North Ryde, NSW 2109, Australia. Email: Pratishtha.chatterjee@mq.edu.au, Telephone: +61-29850-2742.

Professor Ralph N. Martins, Department of Biomedical Sciences, Macquarie University, North Ryde, NSW 2109, Australia. Email: Ralph.martins@mq.edu.au, Telephone: +61-29850-4573.

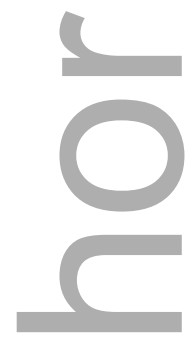

\section{Alphabetical list of abbreviations}

AC: Acylcarnitine

AD: Alzheimer's disease

ADMA: Asymmetric dimethylarginine

ALS: Amyotrophic lateral sclerosis

APOE: Apolipoprotein E genotype 
A $\beta$ : Amyloid- $\beta$

A $\beta$-: Low brain amyloid- $\beta$ load

A $\beta+$ : High brain amyloid- $\beta$ load

BP: Blood pressure

$\mathrm{C}: A P O E \& 4$ carriers

CE: Cholesteryl ester

CKD: Chronic kidney disease

CSF: Cerebrospinal fluid

DASS: Depression, anxiety, stress scales

DG: Diglyceride

FBB: ${ }^{18}$ F-Florbetaben

HRAM-MS: High-resolution accurate mass - mass spectrometer

KARVIAH: Kerr Anglican Retirement Village initiative in ageing health

LLOD: Lower limit of detection

Lyso-PC: Lyso- phospholipid with choline head group

MCI: Mild cognitively impaired

Met-SO: Methionine sulfoxide

MMSE: Mini-mental state examination

MoCA: Montreal cognitive assessment

MRI: Magnetic resonance imaging

NC: $A P O E \varepsilon 4$ non-carriers

NF-L: Neurofilament light chain

NO: Nitric oxide

NOS: Nitric oxide synthase

This article is protected by copyright. All rights reserved 
PC: Phospholipid with choline head group

PD: Parkinson's disease

PET: Positron emission tomography

QC: Quality control

SDMA: Symmetric dimethylarginine

Simoa: Single molecule array

SM: Sphingomyelin

SUVR: Standard uptake value ratio

t4OH-Pro: Trans-4-hydroxyproline

TG: Triglyceride

UHPLC: Ultra-high-performance liquid chromatography

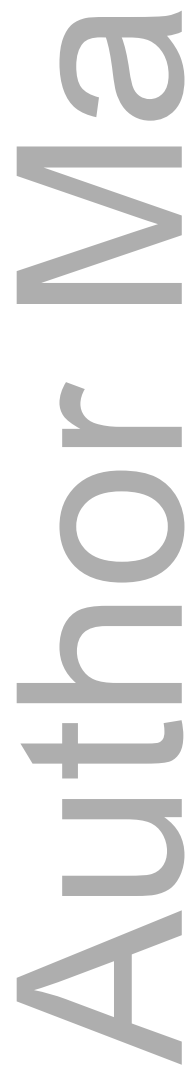




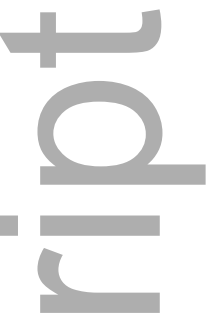

Abstract

Alzheimer's disease (AD) is a progressive neurodegenerative disorder that currently has no cure. Identifying biochemical changes associated with neurodegeneration prior to symptom onset, will provide insight into the biological mechanisms associated with neurodegenerative processes, that may also aid in identifying potential drug targets. The current study therefore investigated associations between plasma neurofilament light chain (NF-L), a marker of neurodegeneration, with plasma metabolites that are products of various cellular processes. Plasma NF-L, measured by ultrasensitive Single molecule array (Simoa) technology (Quanterix) and plasma metabolites, measured by mass-spectrometry (AbsoluteIDQ ${ }^{\circledR} \mathrm{p} 400 \mathrm{HR}$ kit, BIOCRATES), were assessed in the Kerr Anglican Retirement Village Initiative in Ageing Health (KARVIAH) cohort comprising 100 cognitively normal older adults. Metabolites belonging to biogenic amine (creatinine, symmetric dimethylarginine, asymmetric dimethylarginine; ADMA, kynurenine, trans-4-hydroxyproline), amino acid (citrulline, proline, arginine, asparagine, phenylalanine, threonine) and acylcarnitine classes were observed to have positive correlations with plasma NF-L, suggesting a link between neurodegeneration and biological pathways associated with neurotransmitter regulation, nitric oxide homeostasis, inflammation and mitochondrial function. Additionally, after stratifying participants based on low/high brain amyloid- $\beta$ load (A $\beta-/+)$ assessed by positron emission tomography, only ADMA, proline, arginine, asparagine, phenylalanine and acylcarnitine species continued to correlate with NF-L in the A $\beta+$ group, suggesting that the association of these metabolites with neurodegeneration may be relevant to AD-related neuropathology. Metabolites identified to be associated with plasma NF-L may have the potential to serve as prognostic markers for neurodegenerative diseases, however, further studies are required to validate the current findings in an independent cohort, both cross-sectionally and longitudinally. 


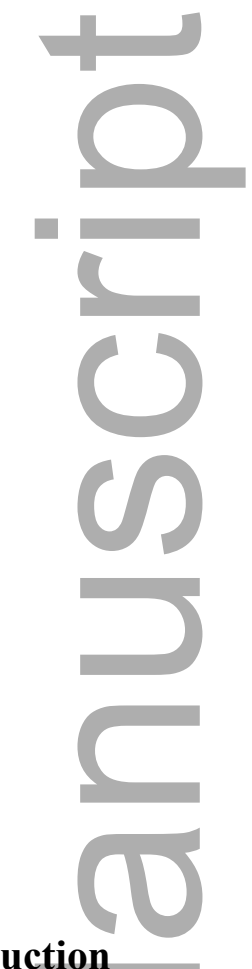

\section{Introduction}

Alzheimer's disease (AD) is a progressive neurodegenerative disorder, characterised by extracellular amyloid- $\beta(\mathrm{A} \beta)$ deposits, intracellular tau aggregates and neuronal loss (Masters et al. 2015).In 2019, nearly 50 million people worldwide were estimated to have AD or another form of dementia and this statistic is expected to escalate to over 150 million by 2050 (Alzheimer's Disease International 2019). Currently AD is incurable, despite significant investments in clinical trials targeted towards effective treatments. Identifying metabolites associated with neurodegeneration prior to symptomatic manifestation of the disease may examine the problem from a different perspective and help to reveal early pathological steps in the cellular mechanisms involved in the AD neurodegenerative process. It may also provide further insight into the identification of potential drug targets for $\mathrm{AD}$ and other neurodegenerative diseases.

Neurofilament light chain (NF-L), an axonal cytoskeletal protein, is increased in the cerebrospinal fluid (CSF) and blood in several neurodegenerative diseases, including AD (Backstrom et al. 2015; Pijnenburg et al. 2015; Scherling et al. 2014; Novakova et al. 2017; Gaiottino et al. 2013; Hansson et al. 2017; Zetterberg et al. 2016; Mattsson et al. 2017). This 
increase has been attributed to the axonal damage occurring in neurodegenerative disorders, resulting in increased neuronal NF-L release into the interstitial fluid with subsequent diffusion into the CSF and blood. Therefore, CSF and blood NF-L levels are increasingly being recognised as features of neurodegeneration.

In $\mathrm{AD}, \mathrm{CSF}$ NF-L concentrations have been reported to correlate with disease pathology and poorer cognitive performance (Zetterberg et al. 2016). Further, blood NF-L concentrations have also been reported to correlate with $\mathrm{AD}$ pathology and cognitive performance in cognitively normal older adults, mild cognitively impaired (MCI) and/or AD individuals (Chatterjee et al. 2018b; Mattsson et al. 2017). Similar findings have been reported in familial AD, where blood NF-L concentrations were higher in pre-symptomatic familial AD individuals, and predicted cognitive changes (Preische et al. 2019; Weston et al. 2017; Weston et al. 2019).

Within the current study, we investigated associations between plasma NF-L, a marker of neurodegeneration, and plasma metabolites that reflect various cellular processes. Additionally, the current study also evaluated associations between plasma NF-L and plasma metabolites separately in individuals at risk of $\mathrm{AD}$, characterised by high brain $\mathrm{A} \beta$ load assessed by positron emission tomography (PET), given that the abnormal build-up of A $\beta$ in the brain begins up to two decades prior to AD symptom onset (Villemagne et al. 2013; Asih et al. 2014). Further, for metabolites that associated with NF-L, correlations were investigated with cognitive performance, hippocampal volume and brain $\mathrm{A} \beta$ load.

\section{Methods}

\section{Participants}

All participants from the Kerr Anglican Retirement Village Initiative in Ageing Health (KARVIAH) met the inclusion and exclusion screening criteria. The inclusion criteria comprised an age range of 65-90 years, good general health and no known significant cerebral vascular disease, fluency in English, adequate or corrected vision and hearing, and no dementia or other pathological cognitive impairment based on a Montreal Cognitive Assessment (MoCA) score $\geq 26$. The study neuropsychologist assessed MoCA scores between 18-25, on a case by case basis, using age and education adjusted norms (Rossetti et al. 2011). The exclusion 
criteria comprised, previous diagnosis of dementia, presence of acute functional psychiatric disorder, history of stroke, depression (based on the depression, anxiety, stress scales; DASS) and uncontrolled hypertension (systolic $\mathrm{BP}>170$ or diastolic $\mathrm{BP}>100$ ). One-hundred and five participants out of the 134 volunteers meeting the inclusion/exclusion criteria underwent blood collection, neuroimaging and neuropsychometric evaluation, while the remaining volunteers either declined neuroimaging or withdrew from the study. Of these 105 participants, 100 were considered cognitively normal based on their Mini Mental State Examination score (MMSE $\geq 26$ ) and were included in the current study. All volunteers provided written informed consent prior to participation and the Bellberry Human Research Ethics Committee, Australia (reference no. 2012-09-1086) and the Macquarie University Human Research Ethics Committee (reference no. 5201701078) provided approval for the study. This study was not pre-registered. This study utilized the entire cohort size available that met the set inclusion/exclusion criteria, and therefore no blinding was required.

\section{Blood collection and APOE genotyping}

Prior to blood withdrawal in the morning, all participants fasted overnight for a minimum of ten hours. The blood drawn was fractionated as described previously (Ellis et al. 2009), and stored at $-80^{\circ} \mathrm{C}$, in 2015 . Apolipoprotein $\mathrm{E}(A P O E)$ genotype was determined from purified genomic DNA extracted from $0.5 \mathrm{ml}$ whole blood as previously described (Goozee et al. 2018).

\section{Measurement of NF-L}

The ultra-sensitive Single molecule array (Simoa) assay platform (Quanterix, Billerica, MA) was employed to measure plasma NF-L concentrations in 2018. All calibrators were run in duplicate. Samples were diluted 4-fold and run in singlicates. Two quality control (QC) samples were run in duplicates at the beginning and end of each plate (Chatterjee et al. 2019). A QC sample with a concentration of $12.1 \mathrm{pg} / \mathrm{mL}$ had repeatability and intermediate precision of $20.2 \%$, and a QC sample with a concentration of $155.8 \mathrm{pg} / \mathrm{mL}$ had repeatability of $14.6 \%$ and intermediate precision of $14.9 \%$.

\section{Measurement of plasma metabolites}

Employing a targeted metabolomics approach, endogenous plasma metabolite concentrations were measured in 2019 using the AbsoluteIDQ ${ }^{\circledR}$ p 400 HR kit (BIOCRATES Life Science AG, Innsbruck, Austria) on an ultra-high-performance liquid chromatography (UHPLC - Thermo Scientific $^{\mathrm{TM}}$ Vanquish $^{\mathrm{TM}}$ ) interfaced with a high-resolution, accurate mass, mass spectrometer 
(HRAM-MS - Thermo Scientific ${ }^{\mathrm{TM}}$ Q Exactive Plus ${ }^{\mathrm{TM}}$ ) platform. The AbsoluteIDQ ${ }^{\circledR}$ p400 HR kit can measure up to 408 endogenous metabolites quantitatively (amino acids and biogenic amines) and semi-quantitatively (acylcarnitines, sphingolipids, choline head group phospholipids, diglycerides and triglycerides) (https://biocrates.com/wpcontent/uploads/2020/02/Biocrates_p400_metabolites.pdf).

Within the current study, plasma samples were de-identified, randomised and blinded prior to being analysed according to the manufacturer's protocol. Test samples were run in singlicates, however a quality control sample (QC2; provided by the manufacturers) was placed after every 19-20 test samples in each kit plate (resulting in five QC2 replicates/plate) to examine consistency in quantitative performance throughout the analysis of each plate and for normalising intra-plate and inter-plate variation (refer to Supplementary Material). Within the current study, metabolites with $>40 \%$ of measurements below the lower limit of detection (LLOD) were excluded from analysis (Toledo et al. 2017). Based on this criteria, 216 metabolite species in the plasma were analysed within the current study, consistent with a report by Thompson and colleagues (Thompson et al. 2019).

\section{Neuropsychological testing}

The MMSE (Folstein et al. 1975) scores were utilised as the primary measure for global cognitive ability assessment within the current study. The MMSE scores range from 0-30, with higher scores indicating better cognitive function and a cut off score below 26 was used for screening out the potential dementia patients. A primary cognitive performance outcome measure was calculated by generating a global composite score for each participant that included verbal and visual episodic memory, as well as, working memory and executive function as described previously (Chatterjee et al. 2018b). The specific neuropsychological tests used to generate the global composite score have been listed in Table 1.

\section{Assessment of brain Aß load via PET}

Participants underwent A $\beta$ PET imaging with ${ }^{18} \mathrm{~F}$-Florbetaben (FBB) and magnetic resonance imaging (MRI) at Macquarie Medical Imaging in Sydney within three months of blood collection. A 4x5 min static scan was acquired $50 \mathrm{~min}$ post intravenous injection of FBB. Brain A $\beta$ load was calculated using CapAIBL (Zhou et al. 2014; Bourgeat et al. 2015) as the mean standard uptake value ratio (SUVR) of the neocortical region including the frontal, superior parietal, lateral temporal, lateral occipital, and anterior and posterior cingulate regions 
normalized to the cerebellar cortex. A cut-off score of 1.35 SUVR was used to categorise participants with low brain $A \beta$ load $(A \beta-, \operatorname{SUVR}<1.35, \mathrm{n}=65)$ and high brain $A \beta$ load $(A \beta+$, $\operatorname{SUVR} \geq 1.35, \mathrm{n}=35$ ). Ninety-six of the 100 participants from the KARVIAH cohort underwent 3D MRI as described previously using a General Electric 3Tesla scanner (Model 750W) (Goozee et al. 2017). Hippocampal volumes were calculated from the T1 MRI images acquired and was normalized to the total intracranial volume comprising the cerebrospinal fluid, grey matter and white matter.

\section{Statistical analyses}

Descriptive statistics including means and standard deviations were calculated for $A \beta$ - and $A \beta+$ groups. Chi-square tests were employed to compare categorical variables between $A \beta$ - and $\mathrm{A} \beta+$ groups. Pearson's correlation coefficient was employed to investigate correlations between plasma NF-L and plasma metabolite concentrations in all participants. Additionally, partial correlations were used to investigate correlations between plasma metabolite concentrations and plasma NF-L after adjustment for covariates, age, sex, $A P O E \varepsilon 4$ carrier status and body mass index (BMI), in all participants. Further, plasma metabolites that correlated with plasma NF-L in all participants, before and after adjusting for covariates, were further investigated for their association with plasma NFL after stratification of participants based on A $\beta-/+$ status, using Pearson's correlation coefficient and partial correlations. For plasma metabolites that correlated with plasma NF-L, Pearson's correlation coefficient and partial correlations, adjusting for the aforementioned covariates, were employed to investigate correlations between plasma metabolite concentrations and cognitive performance, hippocampal volume and brain $\mathrm{A} \beta$ load in all participants. Continuous response variables were natural $\log$ transformed as necessary, to better approximate normality (based on $\mathrm{p}>.05$, Shapiro-Wilk test). No test for outliers was conducted. All participants from the Kerr Anglican Retirement Village Initiative in Ageing Health (KARVIAH) cohort met the inclusion and exclusion screening criteria. Therefore, no sample size calculation was carried out a priori, and no blinding was required - the entire cohort was utilized. All analyses were carried out using IBM $^{\circledR}$ SPSS $^{\circledR}$ Version 23.

\section{Results}

\section{Participant characteristics}


Study participant characteristics including sex, age, BMI, education, $A P O E \& 4$ carriage, MMSE scores, hippocampal volume, plasma NF-L and brain A $\beta$ load are presented in Table 2. On stratifying participants based on $\mathrm{A} \beta-/+$ status, no significant differences in participant characteristics were observed between the two groups, except for a significantly higher $A P O E$ $\varepsilon 4$ carrier frequency in the $\mathrm{A} \beta+$ group compared to the $\mathrm{A} \beta$ - group, as expected (Polvikoski et al. 1995).

\section{Association between AD risk factors (age, sex and $A P O E$ \&4 carriage) and plasma metabolites}

Plasma metabolites observed to have significant associations with age and sex within the KARVIAH cohort are presented in Supplementary Table 1 and Supplementary Table 2, respectively. Briefly, the biogenic amines creatinine, symmetric dimethylarginine (SDMA) and methionine sulfoxide (Met-SO); the amino acids citrulline and proline; acylcarnitine (AC) and lyso-phospholipid with choline head group (lyso-PC) species correlated positively with age. The amino acid tryptophan along with PC, sphingomyelin (SM), cholesteryl ester (CE), diglyceride (DG) and triglyceride (TG) species were observed to correlate inversely with age (Supplementary Table 1). Furthermore, the biogenic amine creatinine; the amino acids, aspartate, glutamic acid, isoleucine, methionine, proline, valine and leucine; and species belonging to the $\mathrm{AC}$, lyso-PC and TG classes were significantly lower in females; whereas the amino acid glycine along with metabolite species within the PC, SM, CE and DG classes were observed to be significantly higher in females (Supplementary Table 2). No significant differences in plasma metabolites were observed between $A P O E \& 4$ non-carriers (NC) and carriers (C), except for DG(44:3) ( $\mathrm{p}=0.030, \mathrm{NC}($ mean $\pm \mathrm{SD}): 2.23 \pm 1.51 \mu \mathrm{M}, \mathrm{C}: 3.07 \pm 1.74 \mu \mathrm{M})$.

\section{Correlation between plasma NF-L and plasma metabolites}

The biogenic amines, creatinine, SDMA, asymmetric dimethylarginine (ADMA), kynurenine and trans-4-hydroxyproline (t4OH-Pro) had positive associations with plasma NF-L levels, both, before and after adjusting for potential confounding variables such as age, sex, APOE $\varepsilon 4$ carriage and BMI in all participants (Table 3, Figure 1A). After stratifying the cohort based on brain $A \beta$ load status $(A \beta-/+)$, creatinine and SDMA continued to correlate with plasma NF-L in both, $A \beta$ - and $A \beta+$ participants, before and after adjusting for the potential confounding variables. Kynurenine correlated significantly with plasma NF-L in $A \beta$ - and $A \beta+$ participants before adjusting for covariates, however, after adjusting for covariates kynurenine correlated 
significantly with plasma NF-L in A $\beta+$ participants and a trend level significance was observed in A $\beta$ - participants. ADMA correlated with plasma NF-L only in A $\beta+$ participants (Table 4).

Additionally, the amino acids citrulline, proline, arginine, asparagine, phenylalanine and threonine were observed to have positive correlations with plasma NF-L, before and after adjusting for covariates (Table 3, Figure 1B). After stratifying the cohort based on brain A $\beta$ load, citrulline correlated with plasma NF-L in both $A \beta$ - and $A \beta+$ participants, while proline, arginine, asparagine and phenylalanine correlated with plasma NF-L only in A $\beta+$ participants (Table 4).

Further, the acylcarnitine species $\mathrm{AC}(0: 0), \mathrm{AC}(2: 0), \mathrm{AC}(8: 1), \mathrm{AC}(10: 0), \mathrm{AC}(10: 1), \mathrm{AC}(10: 2)$, $\mathrm{AC}(10: 3), \mathrm{AC}(12: 1)$ and $\mathrm{AC}(14: 1)$, were observed to have a positive correlation with plasma NF-L levels before and after adjusting for potential confounding variables (Table 3, Figure 1C). After stratifying the cohort based on brain $\mathrm{A} \beta$ load, all the above-mentioned AC species, except $\mathrm{AC}(8: 1)$, correlated significantly with plasma NF-L, in A $\beta+$ participants only (Table 4).

Correlation of cognitive performance, hippocampal volume and brain Aß load with plasma metabolites associated with $N F-L$

The biogenic amines creatinine and SDMA, the amino acids phenylalanine and threonine and the acylcarnitine species $\mathrm{AC}(0: 0), \mathrm{AC}(8: 1), \mathrm{AC}(10: 0), \mathrm{AC}(10: 2), \mathrm{AC}(10: 3), \mathrm{AC}(12: 1)$ and $\mathrm{AC}(14: 1)$, were observed to correlate inversely with the global composite score before adjusting for covariates, however, only threonine and $\mathrm{AC}(12: 1)$ correlated significantly with the global composite score after adjusting for potential confounding factors, age, sex, $A P O E$ $\varepsilon 4$ carriage and BMI (Table 5). Further, creatinine, ADMA and the acylcarnitine species $\mathrm{AC}(3: 0), \mathrm{AC}(8: 1), \mathrm{AC}(10: 2)$ and $\mathrm{AC}(10: 3)$, were observed to correlate inversely with hippocampal volume before adjusting for covariates, while the acylcarnitine species AC(10:0) was observed to correlate positively with brain $A \beta$ load both before and after adjusting for the potential confounding factors (Table 5).

\section{Discussion}

The present study is the first to report on correlations between neurodegeneration, as assessed by the biomarker NF-L, and plasma metabolites representing various cellular processes. Significant associations were found between NF-L and metabolite species belonging to the 
biogenic amine, amino acid and acylcarnitine classes, reflecting a link between neurodegeneration and biological pathways associated with neurotransmitter regulation, nitric oxide homeostasis, inflammation and mitochondrial function. Within the current study, we also found significant correlations between plasma metabolites that associated with biomarker evidence of neurodegeneration, and cognition, hippocampal volume and brain A $\beta$ load.

The biogenic amine creatinine, correlated positively with plasma NF-L, suggesting a positive association between plasma creatinine levels and neurodegeneration. Creatinine was also observed to correlate inversely with cognitive performance and hippocampal volume within the current study. Blood creatinine levels have previously been reported to be higher in AD patients compared to healthy controls (Gonzalez-Dominguez et al. 2014) and also associate inversely with cognitive performance and brain volume (Rajagopalan et al. 2013). Furthermore, higher CSF creatinine levels have been reported in neurodegenerative diseases, such as AD and multiple sclerosis (van der Velpen et al. 2019; Lazzarino et al. 2017). In addition, longitudinal studies have reported that increased blood creatinine levels are associated with an increased risk of cognitive impairment and dementia, and faster rate of cognitive decline (Kurella et al. 2005; Khatri et al. 2009; Etgen et al. 2009; Seliger et al. 2015; Seliger et al. 2004). Our observations within the present study are in line with the above reports. While the exact mechanism behind these associations is not yet understood, a possible neurotoxic effect of creatinine could be mediated by its inhibitory potential towards gamma-aminobutyric acid receptors (De Deyn et al. 2009). Interestingly, in contrast to observations in the current study, lower blood creatinine concentrations have been reported in amyotrophic lateral sclerosis (ALS) patients compared to healthy controls, that also inversely correlated with severity (Lawton et al. 2012; Lawton et al. 2014). These contrasting findings could be attributed to the fact that loss of muscle mass is a feature of ALS and plasma creatinine is a function of muscle mass; while participants within the current study were asymptomatic healthy older adults. Alternatively, as creatinine is a well-known biomarker of renal function, this may reflect the fact that co-morbid renal disease is much less prevalent in ALS (Mitchell et al. 2015).

Plasma levels of the biogenic amines SDMA and ADMA, and the amino acids arginine and citrulline, involved in the nitric oxide (NO) production pathway, correlated positively with biomarker evidence of neurodegeneration, plasma NF-L. Further, SDMA had a significant inverse correlation with cognitive performance while ADMA showed an inverse correlation with cognitive performance with a trend towards statistical significance. Within the NO 
pathway, nitric oxide synthase (NOS) catalyses the conversion of arginine to NO and citrulline. It is well known that NO has several beneficial functions given its antioxidant, anti-apoptotic and vasodilation enhancing properties (Leiper \& Vallance 1999; Dimmeler et al. 1999). However, ADMA has NOS inhibitory properties and SDMA can inhibit cellular arginine uptake, thereby negatively affecting NO production. Therefore, our observations of positive associations of SDMA and ADMA with the plasma neurodegeneration marker were expected. In line with our observations, increased blood ADMA and SDMA levels have previously been reported to be associated with cognitive impairment, neuronal injury and neurodegenerative diseases (McEvoy et al. 2014; Pikula et al. 2009; Luneburg et al. 2012; Arlt et al. 2008; Selley 2003; Kirbas et al. 2016; Javadiyan et al. 2012). The positive associations of plasma arginine and citrulline with NF-L could be attributed to the inhibition of cellular uptake of plasma arginine by SDMA, and consequently, plasma arginine could be converting to plasma citrulline via ornithine (Marini 2012).

Notably, several of the metabolites that positively correlated with NF-L in the present study have also previously been reported as biomarkers of renal function, particularly creatinine and SDMA (Oliva-Damaso et al. 2019; Kielstein et al. 2006; Kassirer 1971; Schmidt \& Baylis 2000). Interestingly, SDMA has been reported as having superior performance compared to other established glomerular filtration rate estimation (eGFR) methods in some populations (Kielstein et al. 2011). This is because almost all SDMA is cleared by renal filtration (Kakimoto \& Akazawa 1970). We found that both creatinine and SDMA had strong associations with NF-L, supporting the possibility of renal dysfunction being associated with neurodegenerative changes. We also observed significant associations between plasma NF-L, and eGFR and urea (Supplementary Table 3), showing associations of NF-L with other renal parameters. It is important to note that the cohort used in this study was in good general health as specified by the inclusion criteria, therefore, individuals with severe kidney or other chronic disease would have been excluded. However, most people with chronic kidney disease (CKD) are asymptomatic until the late stages. Nevertheless, significant associations were also observed between plasma NF-L, and creatinine and SDMA in cohort participants not considered to be at-risk for CKD (based on creatinine levels $\leq 90 \mu \mathrm{M}$ and eGFR $\geq 60$ $\mathrm{mL} / \mathrm{min} / 1.73 \mathrm{~m}^{2}$ ) and in participants reporting no past or present kidney disease (Supplementary Table 4). Results of the current study suggest that plasma metabolites linked to renal dysfunction are associated with biomarker evidence of neurodegeneration, however, the observed correlations in the present study are not attributed to a current kidney disease 
diagnosis. It should also be noted that our knowledge on NF-L clearance from the blood is limited. Whether or not renal clearance is important will be an important topic in future studies. Additionally, plasma kynurenine levels correlated positively with NF-L levels. This was expected, as discussed in detail previously (Chatterjee et al. 2019). The kynurenine pathway is upregulated in neuroinflammatory conditions and, associations of several metabolites from the kynurenine pathway (including kynurenine) with neurodegenerative diseases have been reported previously (Chatterjee et al. 2018a; Chang et al. 2018; Schwarz et al. 2013; Giil et al. 2017; Schwarcz et al. 2012; Lim et al. 2017; Jacobs et al. 2019).

Plasma proline levels were also found to correlate positively with NF-L. In line with this observation, a previous metabolomics study reported higher plasma proline levels in AD patients compared to controls (Wang et al. 2014); and Ibáñez and colleagues reported that CSF proline concentrations (along with other metabolites) could potentially be used to track AD progression (Ibanez et al. 2012). Given that proline has been reported to lower glutamate uptake in rat cerebral cortex in vitro and in vivo (Delwing et al. 2007), it could be posited that the positive association observed between proline and NF-L is attributed to the disruption in glutamate homeostasis with higher proline levels. However, further studies are required to validate this hypothesis.

Plasma levels of asparagine were also observed to have a positive correlation with plasma NFL. Given that asparagine plays a role in the biosynthesis of glycoproteins (N-glycosylation), it could be posited that the positive correlation between asparagine and plasma NF-L may reflect a positive association between the propensity for post-translational modifications and plasma NF-L. The role of glycosylation in AD pathogenesis has been reviewed previously (SchedinWeiss et al 2014). However, in contrast to our observations, CSF asparagine levels have previously been reported to be lower in AD patients compared to controls (Jimenez-Jimenez et al. 1998). Further, Fiandaca and colleagues reported lower mean asparagine plasma levels in individuals with preclinical AD compared to controls (Fiandaca et al. 2015). Therefore, further studies are required to validate the associations observed between plasma asparagine and NFL within the current study.

Additionally, the amino acid phenylalanine was observed to have a positive correlation with NF-L, and a negative correlation with cognitive performance in the present study. In line with our observations, higher plasma phenylalanine concentrations have previously been observed in individuals with amnestic MCI and AD compared to healthy controls (Ravaglia et al. 2004). 
Higher levels of this amino acid have been associated with delirium (Flacker \& Lipsitz 2000; van der Mast et al. 1999). Phenylalanine is a precursor for catecholamines. It has been posited that higher phenylalanine levels may result in brain damage by mechanisms including competition with other large neutral amino acids for transport carriers across the blood brain barrier, decreased protein synthesis in the brain, abnormalities in amine neurotransmitter systems and higher myelin turnover (Surtees \& Blau 2000). Higher threonine levels were reported in advanced Parkinson's disease (PD) patients compared to early PD patients, suggesting an increase in plasma threonine concentrations with PD progression (Figura et al. 2018). These observations are in line with our observations of a positive correlation between threonine and NF-L.

Lastly, within the current study, levels of acylcarnitine species were observed to correlate positively with plasma NF-L. Several acylcarnitine species also had an inverse correlation with cognitive performance and hippocampal volume, and $\mathrm{AC}(10: 0)$ showed a positive correlation with brain A $\beta$ load. Acylcarnitines reportedly have protective neurobiological functions including maintaining synaptic morphology and neurotransmitter signalling (Gorini et al. 1996; Laschi et al. 1990; Bertoni-Freddari et al. 1994; Bertoni-Freddari et al. 1996; Curti et al. 1989; Davis et al. 1993; Piovesan et al. 1995; Imperato et al. 1989) as well as regulating brain energy (Pettegrew et al. 2000; Aureli et al. 1990; Aureli et al. 1994; Rosenthal et al. 1992). Lower blood acylcarnitine concentrations have been reported in individuals with amnestic MCI and AD compared to controls (Mapstone et al. 2014; Cristofano et al. 2016). The positive associations observed between NF-L and acylcarnitine species within the current study are therefore in the opposite direction of what was expected and may reflect a compensatory mechanism within the pre-symptomatic stages of neurodegeneration. However, in line with the current observations, acylcarnitines have also been reported to ameliorate $A \beta$ associated oxidative stress and cell death in vitro (Dhitavat et al. 2002), therefore, further validation studies are required.

Interestingly, after stratifying participants by brain $\mathrm{A} \beta$ status $(\mathrm{A} \beta-/ \mathrm{A} \beta+), \mathrm{ADMA}$, proline, arginine, asparagine, phenylalanine and acylcarnitine species correlated with NF-L only in the $\mathrm{A} \beta+$ group, suggesting that the association of these metabolites with neurodegeneration may be relevant to early AD neuropathology. However, creatinine, SDMA and citrulline correlated with NF-L in both $A \beta$ - and A $\beta+$ individuals. Additionally, kynurenine correlated with NF-L significantly in $A \beta+$ individuals and a trend towards statistical significance $(p=.051)$ was observed in A $\beta$ - participants. These observations may reflect previously reported observations 
of increased plasma or CSF NF-L not being specifically associated with brain A $\beta$ pathology but rather as a feature reflecting both the intensity and the extent of neurodegeneration. For example, elevated NF-L in primary tauopathies with no A $\beta$ pathology, such as multiple system atrophy, progressive supranuclear palsy and corticobasal degeneration have been reported compared to controls (Hansson et al. 2017), suggesting that NF-L concentrations are not driven by brain $A \beta$ load, which likely resulted in the observation of $A \beta-/+$ status independent associations between some plasma metabolites and NF-L. Therefore, such metabolites may serve as markers that may denote neurodegeneration that is not specific to AD.

Limitations acknowledged within the current study include the modest sample size employed, and the cross-sectional nature of the study. Additionally, in comparison to plasma NF-L being employed as a biomarker for neurodegeneration within the current study, CSF NF-L could have served as a more accurate measure of neurodegeneration. However, plasma NF-L has been reported to correlate strongly with CSF NF-L with correlation coefficients $>.75$, showing that plasma NF-L is representative of the axonal disruption manifesting in the CSF (Mattsson et al. 2017; Zetterberg \& Blennow 2018). Another possible limitation is that medication use, and diet were not accounted for in the analyses, which may have affected the outcome. Further, given the modest sample size, adjustments for multiple comparisons were not made as the current study aimed to generate scientific hypotheses that will be critically tested in the future. However, if adjustments for multiple comparisons were to be considered, using Bonferroni correction, $\mathrm{p}<0.00023(0.05 / 216$ metabolites) would be considered significant, leaving the association of creatinine, SDMA and citrulline with NF-L significant even after adjusting for multiple comparisons.

To conclude, the current study identified plasma metabolites that are associated with plasma NF-L, a biomarker for neurodegeneration, providing valuable insights into biological pathways that are triggered in neurodegenerative processes. Additionally, metabolites identified to be associated with plasma NF-L within the current study may have the potential to serve as prognostic markers for neurodegenerative diseases. However, further studies are required to validate the current findings in an independent cohort both cross-sectionally and longitudinally, employing CSF NF-L in parallel with plasma NF-L. Additionally, the specificity of the metabolites for AD would also need to be determined in future studies. 
--Human subjects --

Involves human subjects:

If yes: Informed consent \& ethics approval achieved:

=> if yes, please ensure that the info "Informed consent was achieved for all subjects, and the experiments were approved by the local ethics committee." is included in the Methods

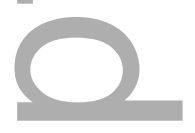

ARRIVE guidelines have been followed:

No

$=>$ if it is a Review or Editorial, skip complete sentence $=>$ if No, include a statement in the "Conflict of interest disclosure" section: "ARRIVE guidelines were not followed for the following reason:

No animals were used in the current study "

(edit phrasing to form a complete sentence as necessary).

$=>$ if Yes, insert in the "Conflict of interest disclosure" section:

"All experiments were conducted in compliance with the ARRIVE guidelines." unless it is a Review or Editorial

Conflicts of interest: HZ has served at scientific advisory boards for Denali, Roche Diagnostics, Wave, Samumed and $\operatorname{CogRx}$, has given lectures in symposia sponsored by Fujirebio, Alzecure and Biogen, and is a co-founder of Brain Biomarker Solutions in Gothenburg AB (BBS), which is a part of the GU Ventures Incubator Program (all outside the submitted work). KB has served as a consultant, at advisory boards, or at data monitoring committees for Abcam, Axon, Biogen, Julius Clinical, Lilly, MagQu, Novartis, Roche Diagnostics, and Siemens Healthineers, and is a co-founder of Brain Biomarker Solutions in Gothenburg AB (BBS), which is a part of the GU Ventures Incubator Program, outside the submitted work.

=> if 'none', insert "The authors have no conflict of interest to declare."

$\Rightarrow>$ otherwise insert info unless it is already included

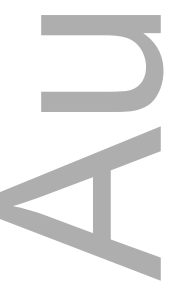

\section{Acknowledgements}

We thank the participants and their families for their participation and cooperation, as well as Anglicare, KaRa Institute of Neurological Diseases and the Australian Alzheimer's Research 
Foundation (AARF) research and support staff for their contributions to this study. We specially thank Ms. Candice ManYan, Ms. Bethany Ball, Ms. Emma Toovey, Ms. Kate Fredericks and Ms. Catherine Brown for their contributions to this study. We also thank the staff of the Macquarie Medical Imaging centre in Macquarie University Hospital, Sydney, for their contributions. We acknowledge the MQ Research Seeding Grant awarded to PC and RNM for funding this project. Florbetaben is a proprietary PET radiopharmaceutical owned by Life Molecular Imaging. For this study, Florbetaben was manufactured and supplied under GMP conditions by Cyclotek (Australia) Pty Ltd. We thank the staff at the Australian Proteome Analysis Facility (APAF) for their support in this study and acknowledge that this study used NCRIS-enabled APAF infrastructure. We also especially thank Dr Manuel Kratzke and Dr Markus Langsdorf for their technical support. KG was a recipient of a grant through the Foundation for Aged Care, Anglicare; and a scholarship from the Co-operative Research Centre for Mental Health. HZ is a Wallenberg Scholar supported by grants from the Swedish Research Council (\#2018-02532), the European Research Council (\#681712), Swedish State Support for Clinical Research (\#ALFGBG-720931), the Alzheimer Drug Discovery Foundation (ADDF), USA (\#201809-2016862), and the UK Dementia Research Institute at UCL. KB is supported by the Swedish Research Council (\#2017-00915), the Alzheimer Drug Discovery Foundation (ADDF), USA (\#RDAPB-201809-2016615), the Swedish Alzheimer Foundation (\#AF-742881), Hjärnfonden, Sweden (\#FO2017-0243), the Swedish state under the agreement between the Swedish government and the County Councils, the ALF-agreement (\#ALFGBG-715986), and European Union Joint Program for Neurodegenerative Disorders (JPND2019-466-236).

\section{Conflicts of interest}

HZ has served at scientific advisory boards for Denali, Roche Diagnostics, Wave, Samumed and $\operatorname{CogRx}$, has given lectures in symposia sponsored by Fujirebio, Alzecure and Biogen, and is a co-founder of Brain Biomarker Solutions in Gothenburg AB (BBS), which is a part of the GU Ventures Incubator Program (all outside the submitted work). KB has served as a consultant, at advisory boards, or at data monitoring committees for Abcam, Axon, Biogen, Julius Clinical, Lilly, MagQu, Novartis, Roche Diagnostics, and Siemens Healthineers, and is a co-founder of Brain Biomarker Solutions in Gothenburg AB (BBS), which is a part of the GU Ventures Incubator Program, outside the submitted work. 


\section{(T) \\ References}

(Alzheimer's Disease International 2019) World Alzheimer Report 2019: Attitudes to dementia. Alzheimer's Disease International.

Arlt, S., Schulze, F., Eichenlaub, M., Maas, R., Lehmbeck, J. T., Schwedhelm, E., Jahn, H. and Boger, R. H. (2008) Asymmetrical dimethylarginine is increased in plasma and decreased in cerebrospinal fluid of patients with Alzheimer's disease. Dement Geriatr Cogn Disord 26, 5864.

Asih, P. R., Chatterjee, P., Verdile, G., Gupta, V. B., Trengove, R. D. and Martins, R. N. (2014) Clearing the amyloid in Alzheimer's: progress towards earlier diagnosis and effective treatments - an update for clinicians. Neurodegenerative disease management 4, 363-378.

Aureli, T., Miccheli, A., Di Cocco, M. E., Ghirardi, O., Giuliani, A., Ramacci, M. T. and Conti, F. (1994) Effect of acetyl-L-carnitine on recovery of brain phosphorus metabolites and lactic acid level during reperfusion after cerebral ischemia in the rat--study by $13 \mathrm{P}$ - and $1 \mathrm{H}-\mathrm{NMR}$ spectroscopy. Brain Res 643, 92-99.

Aureli, T., Miccheli, A., Ricciolini, R., Di Cocco, M. E., Ramacci, M. T., Angelucci, L., Ghirardi, O. and Conti, F. (1990) Aging brain: effect of acetyl-L-carnitine treatment on rat brain energy and phospholipid metabolism. A study by 31P and 1H NMR spectroscopy. Brain Res 526, 108112.

Backstrom, D.C., Eriksson Domellof, M., Linder, J., Olsson, B., Ohrfelt, A., Trupp, M., Zetterberg, H., Blennow, K. and Forsgren, L. (2015) Cerebrospinal Fluid Patterns and the Risk of Future Dementia in Early, Incident Parkinson Disease. JAMA Neurol 72, 1175-1182.

Bertoni-Freddari, C., Fattoretti, P., Caselli, U. and Paoloni, R. (1996) Acetylcarnitine modulation of the morphology of rat hippocampal synapses. Anal Quant Cytol Histol 18, 275-278.

Bertoni-Freddari, C., Fattoretti, P., Casoli, T., Spagna, C. and Casell, U. (1994) Dynamic morphology of the synaptic junctional areas during aging: the effect of chronic acetyl-L-carnitine administration. Brain Res 656, 359-366.

Bourgeat, P., Villemagne, V. L., Dore, V. et al. (2015) Comparison of MR-less PiB SUVR quantification methods. Neurobiol Aging 36 Suppl 1, S159-166. 
Chang, K. H., Cheng, M. L., Tang, H. Y., Huang, C. Y., Wu, Y. R. and Chen, C. M. (2018) Alternations of Metabolic Profile and Kynurenine Metabolism in the Plasma of Parkinson's Disease. Mol Neurobiol 55, 6319-6328.

Chatterjee, P., Goozee, K., Lim, C. K. et al. (2018a) Alterations in serum kynurenine pathway metabolites in individuals with high neocortical amyloid-beta load: A pilot study. Sci Rep 8, 8008

Chatterjee, P., Goozee, K., Sohrabi, H. R. et al. (2018b) Association of Plasma Neurofilament Light Chain with Neocortical Amyloid-beta Load and Cognitive Performance in Cognitively Normal Elderly Participants. J Alzheimers Dis 63, 479-487.

Chatterjee, P., Zetterberg, H., Goozee, K. et al. (2019) Plasma neurofilament light chain and amyloidbeta are associated with the kynurenine pathway metabolites in preclinical Alzheimer's disease. Journal of neuroinflammation 16, 186.

Cristofano, A., Sapere, N., La Marca, G. et al. (2016) Serum Levels of Acyl-Carnitines along the Continuum from Normal to Alzheimer's Dementia. PLoS One 11, e0155694.

Curti, D., Dagani, F., Galmozzi, M. R. and Marzatico, F. (1989) Effect of aging and acetyl-L-carnitine on energetic and cholinergic metabolism in rat brain regions. Mech Ageing Dev 47, 39-45.

Davis, S., Markowska, A. L., Wenk, G. L. and Barnes, C. A. (1993) Acetyl-L-carnitine: behavioral, electrophysiological, and neurochemical effects. Neurobiol Aging 14, 107-115.

De Deyn, P. P., Vanholder, R., Eloot, S. and Glorieux, G. (2009) Guanidino compounds as uremic (neuro)toxins. Semin Dial 22, 340-345.

Delis, D., Kaplan, E. and Kramer, J. (2001) Delis-Kaplan Executive Function System (D-KEFS). San Antonio, TX: The Psychological Corporation.

Delwing, D., Delwing, D., Sanna, R. J., Wofchuk, S. and Wyse, A. T. (2007) Proline promotes decrease in glutamate uptake in slices of cerebral cortex and hippocampus of rats. Life Sci 81, 16451650

Dhitavat, S., Ortiz, D., Shea, T. B. and Rivera, E. R. (2002) Acetyl-L-carnitine protects against amyloidbeta neurotoxicity: roles of oxidative buffering and ATP levels. Neurochem Res 27, 501-505.

Dimmeler, S., Hermann, C., Galle, J. and Zeiher, A. M. (1999) Upregulation of superoxide dismutase and nitric oxide synthase mediates the apoptosis-suppressive effects of shear stress on endothelial cells. Arteriosclerosis, thrombosis, and vascular biology 19, 656-664.

Ellis, K. A., Bush, A. I., Darby, D. et al. (2009) The Australian Imaging, Biomarkers and Lifestyle (AIBL) study of aging: methodology and baseline characteristics of 1112 individuals recruited for a longitudinal study of Alzheimer's disease. Int Psychogeriatr 21, 672-687.

This article is protected by copyright. All rights reserved 
Elwood, R. W. (1991) The Wechsler Memory Scale-Revised: psychometric characteristics and clinical application. Neuropsychology review 2, 179-201.

Estevez-Gonzalez, A., Kulisevsky, J., Boltes, A., Otermin, P. and Garcia-Sanchez, C. (2003) Rey verbal learning test is a useful tool for differential diagnosis in the preclinical phase of Alzheimer's disease: comparison with mild cognitive impairment and normal aging. Int J Geriatr Psychiatry 18, 1021-1028.

Etgen, T., Sander, D., Chonchol, M., Briesenick, C., Poppert, H., Forstl, H. and Bickel, H. (2009) Chronic kidney disease is associated with incident cognitive impairment in the elderly: the INVADE study. Nephrol Dial Transplant 24, 3144-3150.

Fiandaca, M. S., Zhong, X., Cheema, A. K. et al. (2015) Plasma 24-metabolite Panel Predicts Preclinical Transition to Clinical Stages of Alzheimer's Disease. Frontiers in neurology 6, 237.

Figura, M., Kusmierska, K., Bucior, E., Szlufik, S., Koziorowski, D., Jamrozik, Z. and Janik, P. (2018) Serum amino acid profile in patients with Parkinson's disease. PLoS One 13, e0191670.

Flacker, J. M. and Lipsitz, L. A. (2000) Large neutral amino acid changes and delirium in febrile elderly medical patients. The journals of gerontology. Series A, Biological sciences and medical sciences 55, B249-252; discussion B253-244.

Folstein, M. F., Folstein, S. E. and McHugh, P. R. (1975) "Mini-mental state". A practical method for grading the cognitive state of patients for the clinician. J Psychiatr Res 12, 189-198.

Gaiottino, J., Norgren, N., Dobson, R. et al. (2013) Increased neurofilament light chain blood levels in neurodegenerative neurological diseases. PLoS One 8, e75091.

Giil, L. M., Midttun, O., Refsum, H., Ulvik, A., Advani, R., Smith, A. D. and Ueland, P. M. (2017) Kynurenine Pathway Metabolites in Alzheimer's Disease. J Alzheimers Dis 60, 495-504.

Gonzalez-Dominguez, R., Garcia, A., Garcia-Barrera, T., Barbas, C. and Gomez-Ariza, J. L. (2014) Metabolomic profiling of serum in the progression of Alzheimer's disease by capillary electrophoresis-mass spectrometry. Electrophoresis 35, 3321-3330.

Goozee, K., Chatterjee, P., James, I. et al. (2017) Alterations in erythrocyte fatty acid composition in preclinical Alzheimer's disease. Scientific Reports 7, 676.

Goozee, K., Chatterjee, P., James, I. et al. (2018) Elevated plasma ferritin in elderly individuals with high neocortical amyloid-beta load. Mol Psychiatry 23, 1807-1812.

Gorini, A., Ghigini, B. and Villa, R. F. (1996) Acetylcholinesterase activity of synaptic plasma membranes during ageing: effect of L-acetylcarnitine. Dementia 7, 147-154.

Hansson, O., Janelidze, S., Hall, S. et al. (2017) Blood-based NfL: A biomarker for differential diagnosis of parkinsonian disorder. Neurology 88, 930-937.

This article is protected by copyright. All rights reserved 
Ibanez, C., Simo, C., Martin-Alvarez, P. J., Kivipelto, M., Winblad, B., Cedazo-Minguez, A. and Cifuentes, A. (2012) Toward a predictive model of Alzheimer's disease progression using capillary electrophoresis-mass spectrometry metabolomics. Analytical chemistry 84, 85328540 .

Imperato, A., Ramacci, M. T. and Angelucci, L. (1989) Acetyl-L-carnitine enhances acetylcholine release in the striatum and hippocampus of awake freely moving rats. Neurosci Lett 107, 251-255

Jacobs, K. R., Lim, C. K., Blennow, K., Zetterberg, H., Chatterjee, P., Martins, R. N., Brew, B. J., Guillemin, G. J. and Lovejoy, D. B. (2019) Correlation between plasma and CSF concentrations of kynurenine pathway metabolites in Alzheimer's disease and relationship to amyloid-beta and tau. Neurobiol Aging 80, 11-20.

Javadiyan, S., Burdon, K. P., Whiting, M. J., Abhary, S., Straga, T., Hewitt, A. W., Mills, R. A. and Craig, J. E. (2012) Elevation of serum asymmetrical and symmetrical dimethylarginine in patients with advanced glaucoma. Invest Ophthalmol Vis Sci 53, 1923-1927.

Jimenez-Jimenez, F. J., Molina, J. A., Gomez, P. et al. (1998) Neurotransmitter amino acids in cerebrospinal fluid of patients with Alzheimer's disease. J Neural Transm (Vienna) 105, 269277

Kakimoto, Y. and Akazawa, S. (1970) Isolation and identification of N-G,N-G- and N-G,N'-G-dimethylarginine, $\mathrm{N}$-epsilon-mono-, di-, and trimethyllysine, and glucosylgalactosyl- and galactosyldelta-hydroxylysine from human urine. J Biol Chem 245, 5751-5758.

Kassirer, J. P. (1971) Clinical evaluation of kidney function--glomerular function. N Engl J Med 285, 385-389.

Khatri, M., Nickolas, T., Moon, Y. P., Paik, M. C., Rundek, T., Elkind, M. S., Sacco, R. L. and Wright, C. B. (2009) CKD associates with cognitive decline. J Am Soc Nephrol 20, 2427-2432.

Kielstein, J. T., Salpeter, S. R., Bode-Boeger, S. M., Cooke, J. P. and Fliser, D. (2006) Symmetric dimethylarginine (SDMA) as endogenous marker of renal function--a meta-analysis. Nephrol Dial Transplant 21, 2446-2451.

Kielstein, J. T., Veldink, H., Martens-Lobenhoffer, J., Haller, H., Burg, M., Lorenzen, J. M., Lichtinghagen, R., Bode-Boger, S. M. and Kliem, V. (2011) SDMA is an early marker of change in GFR after living-related kidney donation. Nephrol Dial Transplant 26, 324-328.

Kirbas, S., Kirbas, A., Tufekci, A., Cumhur Cure, M., Cakmak, S., Yazici, T. and Cure, E. (2016) Serum levels of homocysteine, asymmetric dimethylarginine and nitric oxide in patients with Parkinson's disease. Acta Clin Belg 71, 71-75.

This article is protected by copyright. All rights reserved 
Kurella, M., Chertow, G. M., Fried, L. F., Cummings, S. R., Harris, T., Simonsick, E., Satterfield, S., Ayonayon, H. and Yaffe, K. (2005) Chronic kidney disease and cognitive impairment in the elderly: the health, aging, and body composition study. J Am Soc Nephrol 16, 2127-2133.

Laschi, R., Badiali de Giorgi, L., Bonvicini, F. and Centurione, L. (1990) Ultrastructural aspects of aging rat hippocampus after long-term administration of acetyl-L-carnitine. Int J Clin Pharmacol Res 10, 59-63.

Lawton, K. A., Brown, M. V., Alexander, D. et al. (2014) Plasma metabolomic biomarker panel to distinguish patients with amyotrophic lateral sclerosis from disease mimics. Amyotrophic lateral sclerosis \& frontotemporal degeneration 15, 362-370.

Lawton, K. A., Cudkowicz, M. E., Brown, M. V. et al. (2012) Biochemical alterations associated with ALS. Amyotroph Lateral Scler 13, 110-118.

Lazzarino, G., Amorini, A. M., Petzold, A., Gasperini, C., Ruggieri, S., Quartuccio, M. E., Lazzarino, G., Di Stasio, E. and Tavazzi, B. (2017) Serum Compounds of Energy Metabolism Impairment Are Related to Disability, Disease Course and Neuroimaging in Multiple Sclerosis. Mol Neurobiol 54, 7520-7533.

Leiper, J. and Vallance, P. (1999) Biological significance of endogenous methylarginines that inhibit nitric oxide synthases. Cardiovascular research 43, 542-548.

Lim, C. K., Bilgin, A., Lovejoy, D. B., Tan, V., Bustamante, S., Taylor, B. V., Bessede, A., Brew, B. J. and Guillemin, G. J. (2017) Kynurenine pathway metabolomics predicts and provides mechanistic insight into multiple sclerosis progression. Sci Rep 7, 41473.

Luneburg, N., von Holten, R. A., Topper, R. F., Schwedhelm, E., Maas, R. and Boger, R. H. (2012) Symmetric dimethylarginine is a marker of detrimental outcome in the acute phase after ischaemic stroke: role of renal function. Clinical science 122, 105-111.

Mapstone, M., Cheema, A. K., Fiandaca, M. S. et al. (2014) Plasma phospholipids identify antecedent memory impairment in older adults. Nat Med.

Marini, J. C. (2012) Arginine and ornithine are the main precursors for citrulline synthesis in mice. J Nutr 142, 572-580.

Masters, C. L., Bateman, R., Blennow, K., Rowe, C. C., Sperling, R. A. and Cummings, J. L. (2015) Alzheimer's disease. Nat Rev Dis Primers 1, 15056.

Mattsson, N., Andreasson, U., Zetterberg, H., Blennow, K. and Alzheimer's Disease Neuroimaging, I. (2017) Association of Plasma Neurofilament Light With Neurodegeneration in Patients With Alzheimer Disease. JAMA Neurol 74, 557-566.

This article is protected by copyright. All rights reserved 
McEvoy, M., Schofield, P., Smith, W., Agho, K., Mangoni, A. A., Soiza, R. L., Peel, R. and Attia, J. (2014) Memory impairment is associated with serum methylarginines in older adults. Curr Alzheimer Res 11, 97-106.

Meyers, J. and Meyers, K. (1995) Rey Complex FigureTest and Recognition Trial. Professional Manual. PsychologicalAssessment Resource, Inc.

Mitchell, C. S., Hollinger, S. K., Goswami, S. D., Polak, M. A., Lee, R. H. and Glass, J. D. (2015) Antecedent Disease is Less Prevalent in Amyotrophic Lateral Sclerosis. Neurodegener Dis 15, 109-113.

Novakova, L., Zetterberg, H., Sundstrom, P. et al. (2017) Monitoring disease activity in multiple sclerosis using serum neurofilament light protein. Neurology 89, 2230-2237.

Oliva-Damaso, E., Oliva-Damaso, N., Rodriguez-Esparragon, F., Payan, J., Baamonde-Laborda, E., Gonzalez-Cabrera, F., Santana-Estupinan, R. and Rodriguez-Perez, J. C. (2019) Asymmetric (ADMA) and Symmetric (SDMA) Dimethylarginines in Chronic Kidney Disease: A Clinical Approach. Int J Mol Sci 20.

Pettegrew, J. W., Levine, J. and McClure, R. J. (2000) Acetyl-L-carnitine physical-chemical, metabolic, and therapeutic properties: relevance for its mode of action in Alzheimer's disease and geriatric depression. Mol Psychiatry 5, 616-632.

Pijnenburg, Y. A., Verwey, N. A., van der Flier, W. M., Scheltens, P. and Teunissen, C. E. (2015) Discriminative and prognostic potential of cerebrospinal fluid phosphoTau/tau ratio and neurofilaments for frontotemporal dementia subtypes. Alzheimers Dement (Amst) 1, 505512.

Pikula, A., Boger, R. H., Beiser, A. S. et al. (2009) Association of plasma ADMA levels with MRI markers of vascular brain injury: Framingham offspring study. Stroke 40, 2959-2964.

Piovesan, P., Quatrini, G., Pacifici, L., Taglialatela, G. and Angelucci, L. (1995) Acetyl-L-carnitine restores choline acetyltransferase activity in the hippocampus of rats with partial unilateral fimbria-fornix transection. Int J Dev Neurosci 13, 13-19.

Polvikoski, T., Sulkava, R., Haltia, M., Kainulainen, K., Vuorio, A., Verkkoniemi, A., Niinisto, L., Halonen, P. and Kontula, K. (1995) Apolipoprotein E, dementia, and cortical deposition of beta-amyloid protein. N Engl J Med 333, 1242-1247.

Preische, O., Schultz, S. A., Apel, A. et al. (2019) Serum neurofilament dynamics predicts neurodegeneration and clinical progression in presymptomatic Alzheimer's disease. Nat Med 25, 277-283. 
Rajagopalan, P., Refsum, H., Hua, X., Toga, A. W., Jack, C. R., Jr., Weiner, M. W., Thompson, P. M. and Alzheimer's Disease Neuroimaging, I. (2013) Mapping creatinine- and cystatin C-related white matter brain deficits in the elderly. Neurobiol Aging 34, 1221-1230.

Ravaglia, G., Forti, P., Maioli, F., Bianchi, G., Martelli, M., Talerico, T., Servadei, L., Zoli, M. and Mariani, E. (2004) Plasma amino acid concentrations in patients with amnestic mild cognitive impairment or Alzheimer disease. Am J Clin Nutr 80, 483-488.

Rosenthal, R. E., Williams, R., Bogaert, Y. E., Getson, P. R. and Fiskum, G. (1992) Prevention of postischemic canine neurological injury through potentiation of brain energy metabolism by acetyl-L-carnitine. Stroke 23, 1312-1317; discussion 1317-1318.

Rossetti, H. C., Lacritz, L. H., Cullum, C. M. and Weiner, M. F. (2011) Normative data for the Montreal Cognitive Assessment (MoCA) in a population-based sample. Neurology 77, 1272-1275.

Schedin-Weiss, S., Winblad, B. and Tjernberg, L. O. (2014) The role of protein glycosylation in Alzheimer disease. FEBS J 281, 46-62.

Scherling, C. S., Hall, T., Berisha, F. et al. (2014) Cerebrospinal fluid neurofilament concentration reflects disease severity in frontotemporal degeneration. Ann Neurol 75, 116-126.

Schmidt, R. J. and Baylis, C. (2000) Total nitric oxide production is low in patients with chronic renal disease. Kidney international 58, 1261-1266.

Schwarcz, R., Bruno, J. P., Muchowski, P. J. and Wu, H. Q. (2012) Kynurenines in the mammalian brain: when physiology meets pathology. Nature reviews. Neuroscience 13, 465-477.

Schwarz, M. J., Guillemin, G. J., Teipel, S. J., Buerger, K. and Hampel, H. (2013) Increased 3hydroxykynurenine serum concentrations differentiate Alzheimer's disease patients from controls. Eur Arch Psychiatry Clin Neurosci 263, 345-352.

Seliger, S. L., Siscovick, D. S., Stehman-Breen, C. O., Gillen, D. L., Fitzpatrick, A., Bleyer, A. and Kuller, L. H. (2004) Moderate renal impairment and risk of dementia among older adults: the Cardiovascular Health Cognition Study. J Am Soc Nephrol 15, 1904-1911.

Seliger, S. L., Wendell, C. R., Waldstein, S. R., Ferrucci, L. and Zonderman, A. B. (2015) Renal function and long-term decline in cognitive function: the Baltimore Longitudinal Study of Aging. Am J Nephrol 41, 305-312.

Selley, M. L. (2003) Increased concentrations of homocysteine and asymmetric dimethylarginine and decreased concentrations of nitric oxide in the plasma of patients with Alzheimer's disease. Neurobiol Aging 24, 903-907.

Surtees, R. and Blau, N. (2000) The neurochemistry of phenylketonuria. Eur J Pediatr 159 Suppl 2, S109-113.

This article is protected by copyright. All rights reserved 
Thompson, J. W., Adams, K. J., Adamski, J. et al. (2019) International Ring Trial of a High Resolution Targeted Metabolomics and Lipidomics Platform for Serum and Plasma Analysis. Analytical chemistry 91, 14407-14416.

Toledo, J. B., Arnold, M., Kastenmuller, G. et al. (2017) Metabolic network failures in Alzheimer's disease: A biochemical road map. Alzheimers Dement 13, 965-984.

van der Mast, R. C., van den Broek, W. W., Fekkes, D., Pepplinkhuizen, L. and Habbema, J. D. (1999) Incidence of and preoperative predictors for delirium after cardiac surgery. $J$ Psychosom Res 46, 479-483.

van der Velpen, V., Teav, T., Gallart-Ayala, H. et al. (2019) Systemic and central nervous system metabolic alterations in Alzheimer's disease. Alzheimers Res Ther 11, 93.

Villemagne, V.L., Burnham, S., Bourgeat, P. et al. (2013) Amyloid beta deposition, neurodegeneration, and cognitive decline in sporadic Alzheimer's disease: a prospective cohort study. Lancet Neurol 12, 357-367.

Wang, G., Zhou, Y., Huang, F. J. et al. (2014) Plasma metabolite profiles of Alzheimer's disease and mild cognitive impairment. J Proteome Res 13, 2649-2658.

Wechsler, D. (1997) Wechsler Adult Intelligence Scale - III (WAIS-III) Administration and Scoring Manual., The Psychological Corporation, San Antonio, TX.

Wechsler, D. A. (1987) Wechsler memory scale-revised. , Psychological Corporation, New York. Weston, P. S. J., Poole, T., O'Connor, A. et al. (2019) Longitudinal measurement of serum neurofilament light in presymptomatic familial Alzheimer's disease. Alzheimers Res Ther 11, 19.

Weston, P. S. J., Poole, T., Ryan, N. S. et al. (2017) Serum neurofilament light in familial Alzheimer disease: A marker of early neurodegeneration. Neurology 89, 2167-2175.

Zetterberg, H. and Blennow, K. (2018) From Cerebrospinal Fluid to Blood: The Third Wave of Fluid Biomarkers for Alzheimer's Disease. J Alzheimers Dis 64, S271-S279.

Zetterberg, H., Skillback, T., Mattsson, N., Trojanowski, J. Q., Portelius, E., Shaw, L. M., Weiner, M. W., Blennow, K. and Alzheimer's Disease Neuroimaging, I. (2016) Association of Cerebrospinal Fluid Neurofilament Light Concentration With Alzheimer Disease Progression. JAMA Neurol 73, 60-67.

Zhou, L., Salvado, O., Dore, V. et al. (2014) MR-less surface-based amyloid assessment based on $11 \mathrm{C}$ PiB PET. PLoS One 9, e84777. 


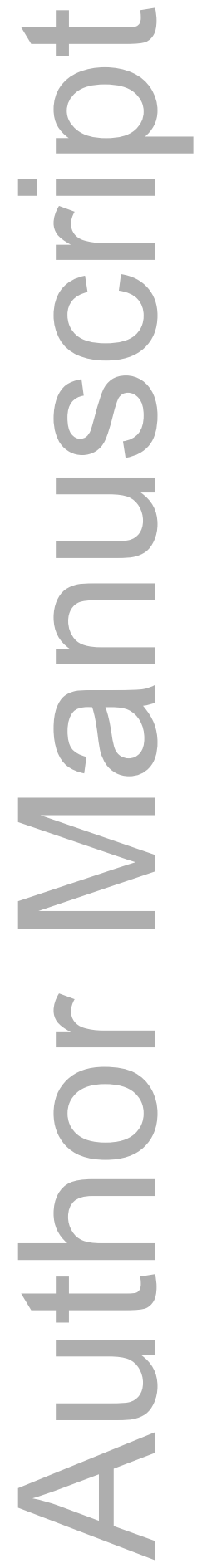

This article is protected by copyright. All rights reserved 


\section{Tables}

Table 1. List of neuropsychological tests used to generate the global composite score. The global composite score was generated from the mean of the z-score measures of the tests listed below.

\begin{tabular}{|l|l|}
\hline Test & Reference \\
\hline $\begin{array}{l}\text { Rey Auditory Verbal Learning Test (RAVLT) List A, short delay, long } \\
\text { delay }\end{array}$ & $\begin{array}{l}\text { Estevez- } \\
\text { Gonzalez et al. } \\
2003)\end{array}$ \\
\hline Logical Memory (LM) I and II (WMS-III; Story A only) & (Elwood 1991) \\
\hline Rey Complex Figure Test (RCFT) 3 min and RCFT 30 min & $\begin{array}{l}\text { (Meyers \& } \\
\text { Meyers 1995) }\end{array}$ \\
\hline $\begin{array}{l}\text { Wechsler Adult Intelligence Scale - Third edition (WAIS-III) Digit Span } \\
\text { backward (Wechsler } \\
\text { WAIS-III Digit Symbol Substitution Test (DSST) }\end{array}$ & $\begin{array}{l}\text { (Wechsler } \\
1997)\end{array}$ \\
\hline $\begin{array}{l}\text { D-KEFS Category Fluency (Boys Names) and Switching (Fruits and } \\
\text { Furniture) Tasks }\end{array}$ & $\begin{array}{l}\text { (Delis et al. } \\
2001)\end{array}$ \\
\hline MMSE (Folstein et al. \\
$1975)$
\end{tabular}




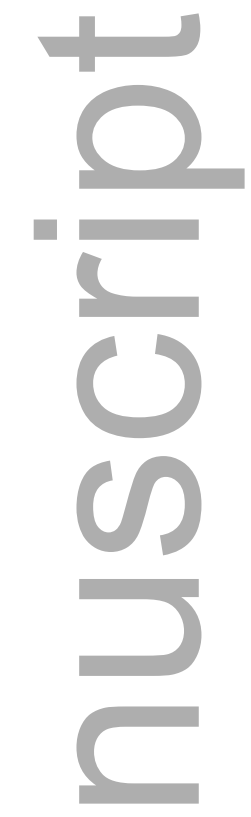

Table 2. Study participant characteristics. Participant characteristics including sex, age, body mass index (BMI), education, $A P O E \& 4$ status, mini mental state examination (MMSE) score, global composite score, hippocampal volume (HV; normalised with intracranial volume), neurofilament light chain concentrations (NF-L) and brain A $\beta$ load (represented by the standard uptake value ratio (SUVR) of the ligand ${ }^{18} \mathrm{~F}$-Florbetaben in the neocortical region normalised with that in the cerebellum) are presented for all participants, and stratified by brain $A \beta$ load status, i.e. $A \beta-(S U V R<1.35)$ and $A \beta+(S U V R \geq 1.35)$. Chi-square tests or linear models were employed, as appropriate. $\mathrm{p}$ represents $\mathrm{p}$-values for comparison of participant characteristics between $A \beta$ - and $A \beta+$ groups. $\mathrm{p}<0.05$ was considered significant and is presented in bold font. $\mathrm{N}$ represents the number of participants.

\begin{tabular}{|c|c|c|c|c|}
\hline 7 & All participants & $\mathrm{A} \beta-$ & $\mathrm{A} \beta+$ & $\mathrm{p}$ \\
\hline $\mathrm{N}$ & 100 & 65 & 35 & - \\
\hline $\operatorname{Sex}(M / F)$ & $32 / 68$ & $19 / 46$ & $13 / 22$ & 0.419 \\
\hline Age (years, mean \pm SD) & $78.18 \pm 5.52$ & $77.62 \pm 5.56$ & $79.23 \pm 5.38$ & 0.165 \\
\hline $\mathrm{BMI}\left(\mathrm{kg} / \mathrm{m}^{2}\right.$, mean $\left.\pm \mathrm{SD}\right)$ & $27.62 \pm 4.56$ & $27.39 \pm 4.48$ & $28.06 \pm 4.74$ & 0.486 \\
\hline Education (years, mean \pm SD) & $14.43 \pm 3.26$ & $14.85 \pm 3.37$ & $13.64 \pm 2.92$ & 0.078 \\
\hline
\end{tabular}




\begin{tabular}{|c|c|c|c|c|}
\hline $\mathrm{n} A P O E \& 4$ carriers $(\%)$ & $21(21)$ & $5(7.69)$ & $16(45.71)$ & $<0.0001$ \\
\hline MMSE (mean \pm SD) & $28.61 \pm 1.15$ & $28.51 \pm 1.16$ & $28.80 \pm 1.11$ & 0.225 \\
\hline Global composite score (mean \pm SD) & $0.00 \pm 0.55$ & $0.07 \pm 0.49$ & $-.13 \pm 0.63$ & 0.080 \\
\hline $\mathrm{HV} \%$ (mean $\pm \mathrm{SD}, \mathrm{n}=96$, left and & $0.195 \pm 0.02$ & $0.195 \pm 0.0201$ & $0.194 \pm 0.0197$ & 0.805 \\
\hline right lobes) & $0.199 \pm 0.0204$ & $0.199 \pm 0.0214$ & $0.200 \pm 0.0186$ & 0.891 \\
\hline NF-L (pg/mL, mean \pm SD) & $36.38 \pm 17.78$ & $35.24 \pm 17.28$ & $38.50 \pm 18.76$ & 0.384 \\
\hline Brain $A \beta$ load (SUVR, mean \pm SD) & $1.35 \pm 0.32$ & $1.16 \pm 0.09$ & $1.71 \pm 0.26$ & - \\
\hline
\end{tabular}

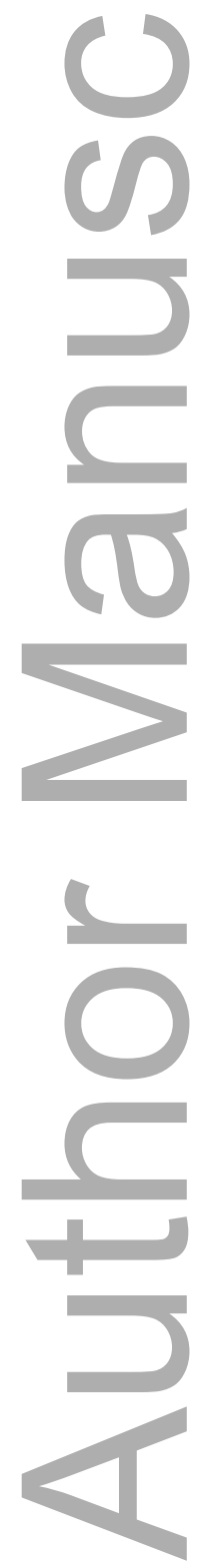


Table 3. Plasma metabolites correlating with plasma neurofilament light chain. Correlations were investigated using Pearson's correlation coefficient (r) and partial correlations ( $\left.\mathrm{r}^{\mathrm{a}}\right)$, adjusting for age, sex, $A P O E \& 4$ carrier status and body mass index (BMI), in all participants. Metabolites significantly correlating with NF-L both before and after adjusting for covariates have been reported. $\dagger$ represents metabolites that were natural log transformed to better approximate normal distribution. SDMA: symmetric dimethylarginine, ADMA: asymmetric dimethylarginine, t4OH-Pro: trans-4hydroxyproline, AC: acylcarnitine.

\begin{tabular}{|c|c|c|c|c|}
\hline Analyte & $\mathrm{r}$ & $\mathrm{p}$ & $\mathrm{r}^{\mathrm{a}}$ & $\mathrm{p}$ \\
\hline \multicolumn{5}{|l|}{ Biogenic amines } \\
\hline Creatinine & .544 & 0.000000005 & .558 & 0.000000003 \\
\hline $\mathrm{SDMA} \dagger$ & .560 & 0.000000001 & .503 & 0.0000002 \\
\hline ADMA & .250 & 0.012 & .228 & 0.026 \\
\hline Kynurenin & .315 & 0.001 & .358 & 0.0003 \\
\hline t4OH-Pro & .243 & 0.015 & .221 & $\mathbf{0 . 0 3 1}$ \\
\hline \multicolumn{5}{|l|}{ Amino acids } \\
\hline Citrulline & .428 & 0.000009 & .390 & 0.00009 \\
\hline Proline $†$ & .297 & 0.003 & .260 & 0.010 \\
\hline Arginine & .233 & 0.020 & .296 & 0.003 \\
\hline Asparagine & .271 & 0.006 & .251 & 0.014 \\
\hline Phenylalanine & .210 & 0.036 & .215 & 0.036 \\
\hline Threonine & .253 & 0.011 & .208 & 0.042 \\
\hline \multicolumn{5}{|l|}{ Acylcarnitines } \\
\hline $\mathrm{AC}(0: 0)$ & .294 & 0.003 & .288 & 0.004 \\
\hline $\mathrm{AC}(2: 0)$ & .248 & 0.013 & .260 & 0.011 \\
\hline $\mathrm{AC}(8: 1) \dagger$ & .348 & 0.0004 & .207 & 0.043 \\
\hline $\mathrm{AC}(10: 0)$ & .370 & 0.0001 & .356 & 0.0004 \\
\hline $\mathrm{AC}(10: 1)$ & .343 & 0.0005 & .296 & 0.003 \\
\hline $\mathrm{AC}(10: 2)$ & .417 & 0.00002 & .289 & 0.004 \\
\hline $\mathrm{AC}(10: 3)$ & .401 & 0.00003 & .295 & 0.004 \\
\hline $\mathrm{AC}(12: 1)$ & .364 & 0.0002 & .343 & 0.001 \\
\hline $\mathrm{AC}(14: 1) \dagger$ & .338 & 0.001 & .267 & 0.009 \\
\hline
\end{tabular}




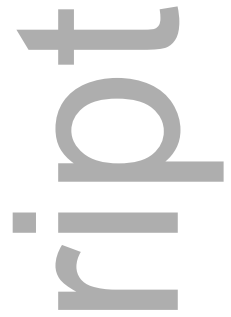

Table 4. Plasma metabolites correlating with plasma neurofilament light chain, in participants stratified by $\mathbf{A} \boldsymbol{\beta}$ status. Correlations were investigated using Pearson's correlation coefficient (r) and partial correlations $\left(\mathrm{r}^{\mathrm{a}}\right)$, adjusting for age, sex, $A P O E \& 4$ carrier status and body mass index (BMI). Participants were categorised as $A \beta-/+$ based on their brain $A \beta$ load measured via positron emission tomography using ligand ${ }^{18} \mathrm{~F}$-Florbetaben. $\dagger$ represents metabolites that were natural log transformed to better approximate normal distribution. SDMA: symmetric dimethylarginine, ADMA: asymmetric dimethylarginine, t4OH-Pro: trans-4-hydroxyproline, $\mathrm{AC}$ : acylcarnitine. $\mathrm{p}<0.05$ was considered significant and is presented in bold font.

\begin{tabular}{|c|c|c|c|c|c|c|c|c|}
\hline & \multicolumn{4}{|c|}{$\mathbf{A \beta}-$} & \multicolumn{4}{|c|}{$\mathbf{A} \boldsymbol{\beta}+$} \\
\hline Analyte & $r$ & $\mathrm{p}$ & $\mathrm{r}^{\mathrm{a}}$ & $\mathrm{p}$ & $\mathrm{r}$ & $\mathrm{p}$ & $\mathrm{r}^{\mathrm{a}}$ & $\mathrm{p}$ \\
\hline \multicolumn{9}{|c|}{ Biogenic amines } \\
\hline Creatinine & .506 & 0.00002 & .506 & 0.00003 & .610 & 0.0001 & .627 & 0.0002 \\
\hline $\mathrm{SDMA} \dagger$ & .527 & 0.00001 & .439 & 0.0004 & .610 & 0.0001 & .638 & 0.0001 \\
\hline ADMA & .170 & 0.177 & .140 & 0.282 & .368 & 0.030 & .357 & 0.049 \\
\hline Kynurenine & .265 & 0.033 & .251 & 0.051 & .395 & 0.019 & .560 & 0.001 \\
\hline t4OH-Pro $t$ & .153 & 0.224 & .177 & 0.173 & .431 & 0.010 & .324 & 0.075 \\
\hline \multicolumn{9}{|c|}{ Amino acids } \\
\hline Citrulline $\dagger$ & .380 & 0.002 & .305 & 0.017 & .506 & 0.002 & .590 & 0.0005 \\
\hline Proline $\dagger$ & .193 & 0.124 & .108 & 0.406 & .434 & 0.009 & .473 & 0.007 \\
\hline Arginin & .151 & 0.229 & .223 & 0.084 & .350 & 0.039 & .482 & 0.006 \\
\hline Asparagine & .139 & 0.268 & .127 & 0.330 & .459 & 0.005 & .441 & 0.013 \\
\hline Phenylalanine & .129 & 0.307 & .073 & 0.575 & .320 & 0.061 & .450 & 0.011 \\
\hline Threonine & .101 & 0.424 & .171 & 0.189 & .448 & 0.007 & .264 & 0.152 \\
\hline Acylcarnitines & & & & & & & & \\
\hline
\end{tabular}




\begin{tabular}{|l|ll|ll|ll|ll|}
$\mathrm{AC}(0: 0)$ & .221 & 0.077 & .186 & 0.152 & .406 & $\mathbf{0 . 0 1 6}$ & .587 & $\mathbf{0 . 0 0 1}$ \\
$\mathrm{AC}(2: 0)$ & .227 & 0.069 & .140 & 0.282 & .310 & 0.070 & .580 & $\mathbf{0 . 0 0 1}$ \\
$\mathrm{AC}(8: 1) \dagger$ & .299 & $\mathbf{0 . 0 1 6}$ & .169 & 0.193 & .457 & $\mathbf{0 . 0 0 6}$ & .319 & 0.080 \\
$\mathrm{AC}(10: 0)$ & .318 & $\mathbf{0 . 0 1 0}$ & .251 & 0.051 & .437 & $\mathbf{0 . 0 0 9}$ & .631 & $\mathbf{0 . 0 0 0 1}$ \\
$\mathrm{AC}(10: 1)$ & .264 & $\mathbf{0 . 0 3 4}$ & .153 & 0.240 & .455 & $\mathbf{0 . 0 0 6}$ & .580 & $\mathbf{0 . 0 0 1}$ \\
$\mathrm{AC}(10: 2)+$ & .372 & $\mathbf{0 . 0 0 2}$ & .236 & 0.067 & .517 & $\mathbf{0 . 0 0 1}$ & .371 & $\mathbf{0 . 0 4 0}$ \\
$\mathrm{AC}(10: 3) \dagger$ & .330 & $\mathbf{0 . 0 0 7}$ & .182 & 0.160 & .542 & $\mathbf{0 . 0 0 1}$ & .476 & $\mathbf{0 . 0 0 7}$ \\
$\mathrm{AC}(12: 1)$ & .309 & $\mathbf{0 . 0 1 2}$ & .224 & 0.083 & .431 & $\mathbf{0 . 0 1 0}$ & .596 & $\mathbf{0 . 0 0 0 4}$ \\
$\mathrm{AC}(14: 1) \dagger$ & .290 & $\mathbf{0 . 0 1 9}$ & .139 & 0.286 & .418 & $\mathbf{0 . 0 1 2}$ & .620 & $\mathbf{0 . 0 0 0 2}$ \\
\hline
\end{tabular}

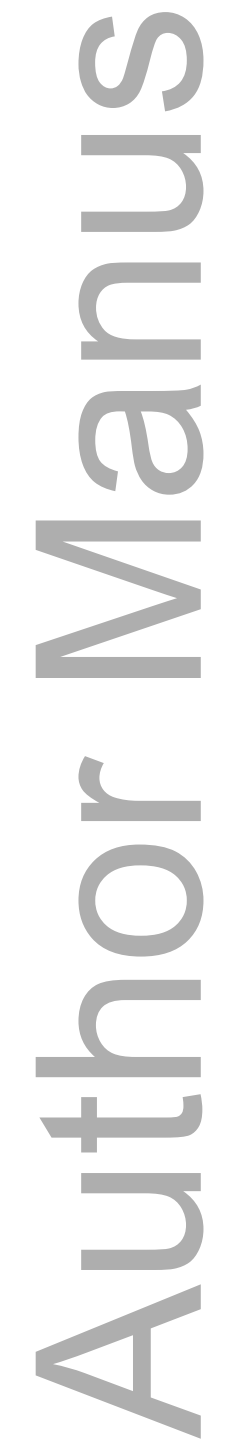


Table 5. Association of cognitive performance, hippocampal volume and brain A $\beta$ load with plasma metabolites correlating significantly with plasma

NF-L. Correlations were investigated using Pearson's correlation coefficient (r) and partial correlations ( $\left.\mathrm{r}^{\mathrm{a}}\right)$, adjusting for age, sex, $A P O E$ \& 4 carrier status and body mass index (BMI) in all participants. Cognitive performance was calculated by generating a global composite score as described in the Methods section. $\dagger$ represents metabolites that were natural log transformed to better approximate normal distribution. SDMA: symmetric dimethylarginine, ADMA: asymmetric dimethylarginine, t4OH-Pro: trans-4-hydroxyproline, AC: acylcarnitine. $\mathrm{p}<0.05$ was considered significant.

\begin{tabular}{|c|c|c|c|c|c|c|c|c|c|c|c|c|c|c|c|c|}
\hline & \multicolumn{4}{|c|}{ Cognitive performance } & \multicolumn{4}{|c|}{ Left hippocampus volume } & \multicolumn{4}{|c|}{ Right hippocampus volume } & \multicolumn{4}{|c|}{ Brain A $\beta$ load } \\
\hline Analyte & $\mathrm{r}$ & $\mathrm{p}$ & $\mathrm{r}^{\mathrm{a}}$ & $\mathrm{p}$ & $\mathrm{r}$ & $\mathrm{p}$ & $\mathrm{r}^{\mathrm{a}}$ & $\mathrm{p}$ & $\mathrm{r}$ & $\mathrm{p}$ & $\mathrm{r}^{\mathrm{a}}$ & $\mathrm{p}$ & $\mathrm{r}$ & $\mathrm{p}$ & $\mathrm{r}^{\mathrm{a}}$ & $\mathrm{p}$ \\
\hline \multicolumn{17}{|c|}{ Biogenic amines } \\
\hline Creatinine + & -.348 & 0.0004 & -.156 & 0.130 & -.226 & 0.027 & -.081 & 0.441 & -.202 & 0.049 & -.038 & 0.721 & .140 & 0.165 & .067 & 0.516 \\
\hline SDMA & -.258 & 0.009 & -.115 & 0.263 & -.162 & 0.114 & -.055 & 0.600 & -.178 & 0.082 & -.059 & 0.578 & .151 & 0.133 & .074 & 0.476 \\
\hline ADMA & -.170 & 0.092 & -.136 & 0.186 & -.064 & 0.535 & -.021 & 0.840 & -.210 & 0.040 & -.162 & 0.122 & .062 & 0.542 & .039 & 0.706 \\
\hline Kynurenine $\dagger$ & -.091 & 0.369 & -.039 & 0.706 & -.126 & 0.223 & -.081 & 0.444 & -.170 & 0.097 & -.109 & 0.302 & .113 & 0.263 & .053 & 0.610 \\
\hline $\mathrm{t} 4 \mathrm{OH}-\mathrm{Pro} \dagger$ & -.168 & 0.094 & -.144 & 0.162 & -.082 & 0.426 & -.054 & 0.611 & -.028 & 0.785 & .022 & 0.837 & -.027 & 0.787 & -.028 & 0.787 \\
\hline \multicolumn{17}{|l|}{ Amino acids } \\
\hline Citrulline + & -.153 & 0.128 & -.051 & 0.623 & -.092 & 0.375 & -.021 & 0.842 & -.080 & 0.439 & -.002 & 0.981 & -.006 & 0.956 & -.010 & 0.923 \\
\hline Proline $\uparrow$ & -.170 & 0.090 & -.042 & 0.682 & -.124 & 0.228 & -.015 & 0.885 & -.163 & 0.114 & -.032 & 0.764 & .149 & 0.140 & -.002 & 0.984 \\
\hline Arginine & -.149 & 0.139 & -.153 & 0.138 & .006 & 0.953 & .017 & 0.871 & -.104 & 0.312 & -.101 & 0.339 & .046 & 0.652 & .062 & 0.550 \\
\hline Asparagine & -.137 & 0.175 & -.096 & 0.354 & -.094 & 0.365 & -.062 & 0.559 & -.113 & 0.272 & -.085 & 0.422 & .049 & 0.629 & .070 & 0.496 \\
\hline Phenylalanine & -.220 & 0.028 & -.173 & 0.092 & -.044 & 0.670 & .014 & 0.894 & -.087 & 0.401 & -.022 & 0.837 & .089 & 0.379 & .050 & 0.629 \\
\hline Threonine & -.266 & 0.008 & -.227 & 0.026 & -.142 & 0.167 & -.104 & 0.325 & -.112 & 0.276 & -.066 & 0.529 & .042 & 0.675 & .042 & 0.684 \\
\hline \multicolumn{17}{|l|}{ Acylcarnitines } \\
\hline $\mathrm{AC}(0: 0)$ & -.238 & 0.017 & -.178 & 0.082 & -.116 & 0.261 & -.055 & 0.601 & -.197 & 0.055 & -.131 & 0.214 & .159 & 0.115 & .133 & 0.198 \\
\hline
\end{tabular}

This article is protected by copyright. All rights reserved 


\begin{tabular}{|c|c|c|c|c|c|c|c|c|c|c|c|c|c|c|c|c|}
\hline $\mathrm{AC}(2: 0)$ & -.143 & 0.157 & -.075 & 0.467 & -.153 & 0.136 & -.097 & 0.355 & -.191 & 0.062 & -.133 & 0.207 & .015 & 0.883 & -.012 & 0.904 \\
\hline $\mathrm{AC}(8: 1) \dagger$ & -.222 & 0.027 & -.083 & 0.424 & -.272 & 0.007 & -.182 & 0.083 & -.279 & 0.006 & -.172 & 0.100 & .160 & 0.112 & .123 & 0.232 \\
\hline $\mathrm{AC}(10:$ & -.231 & 0.021 & -.155 & 0.131 & -.166 & 0.107 & -.106 & 0.313 & -.102 & 0.324 & -.041 & 0.701 & .221 & 0.027 & .229 & 0.025 \\
\hline $\mathrm{AC}(10: 1)$ & -.179 & 0.075 & -.082 & 0.426 & -.099 & 0.336 & -.032 & 0.760 & -.053 & 0.605 & .011 & 0.917 & .125 & 0.214 & .164 & 0.109 \\
\hline $\mathrm{AC}(10: 2) \dagger$ & -.258 & 0.009 & -.121 & 0.240 & -.223 & 0.029 & -.135 & 0.200 & -.216 & 0.034 & -.123 & 0.243 & .073 & 0.468 & .089 & 0.388 \\
\hline $\mathrm{AC}(10: 3)$ & -.198 & 0.048 & -.028 & 0.783 & -.280 & 0.006 & -.180 & 0.085 & -.257 & 0.011 & -.148 & 0.160 & .100 & 0.321 & .057 & 0.583 \\
\hline $\mathrm{AC}(12$ & -.283 & 0.004 & -.237 & 0.020 & -.145 & 0.158 & -.105 & 0.321 & -.145 & 0.159 & -.114 & 0.280 & .192 & 0.055 & .174 & 0.090 \\
\hline $\mathrm{AC}(14: 1) \dagger$ & -.259 & 0.009 & -.180 & 0.079 & -.083 & 0.422 & -.006 & 0.955 & -.151 & 0.142 & -.075 & 0.476 & .155 & 0.123 & .124 & 0.229 \\
\hline
\end{tabular}

This article is protected by copyright. All rights reserved 


\section{Figures}

Figure 1. Correlation between plasma NF-L and plasma metabolites in all participants. Correlation of plasma NF-L with A. Biogenic amines, creatinine, symmetric dimethylarginine (SDMA), asymmetric dimethylarginine (ADMA), kynurenine and trans-4-hydroxy proline (t4OH-Pro), B. Amino acids, citrulline, proline, arginine, asparagine, threonine and phenylalanine and C. Acylcarnitine species using Pearson's correlation coefficient (r). Concentrations of plasma NF-L and metabolites (as necessary) were natural log transformed to better approximate normality. $\mathrm{P}<.05$ was considered significant. Number of participants $=$ 100.

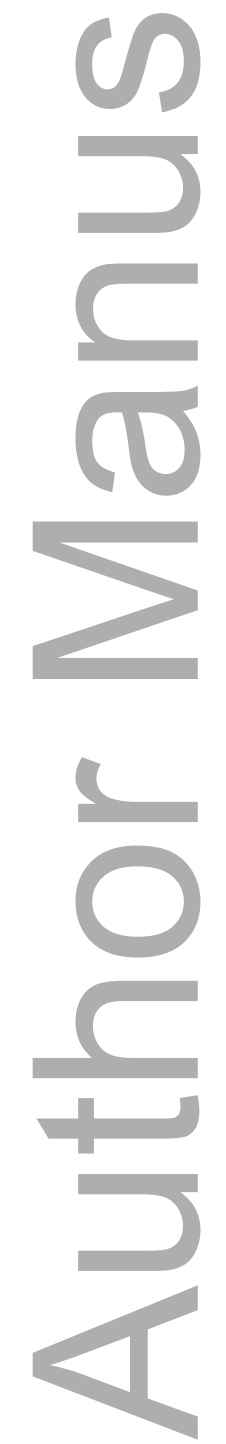




\section{Figures}

Figure 1. Correlation between plasma NF-L and plasma metabolites in all participants. Correlation of plasma NF-L with A. Biogenic amines, creatinine, symmetric dimethylarginine (SDMA), asymmetric dimethylarginine (ADMA), kynurenine and trans-4-hydroxy proline (t4OH-Pro), B. Amino acids, citrulline, proline, arginine, asparagine, threonine and phenylalanine and C. Acylcarnitine species using Pearson's correlation coefficient (r). Concentrations of plasma NF-L and metabolites (as necessary) were natural log transformed to better approximate normality. $\mathrm{P}<.05$ was considered significant. Number of participants = 100 .

\section{A. Biogenic amines}
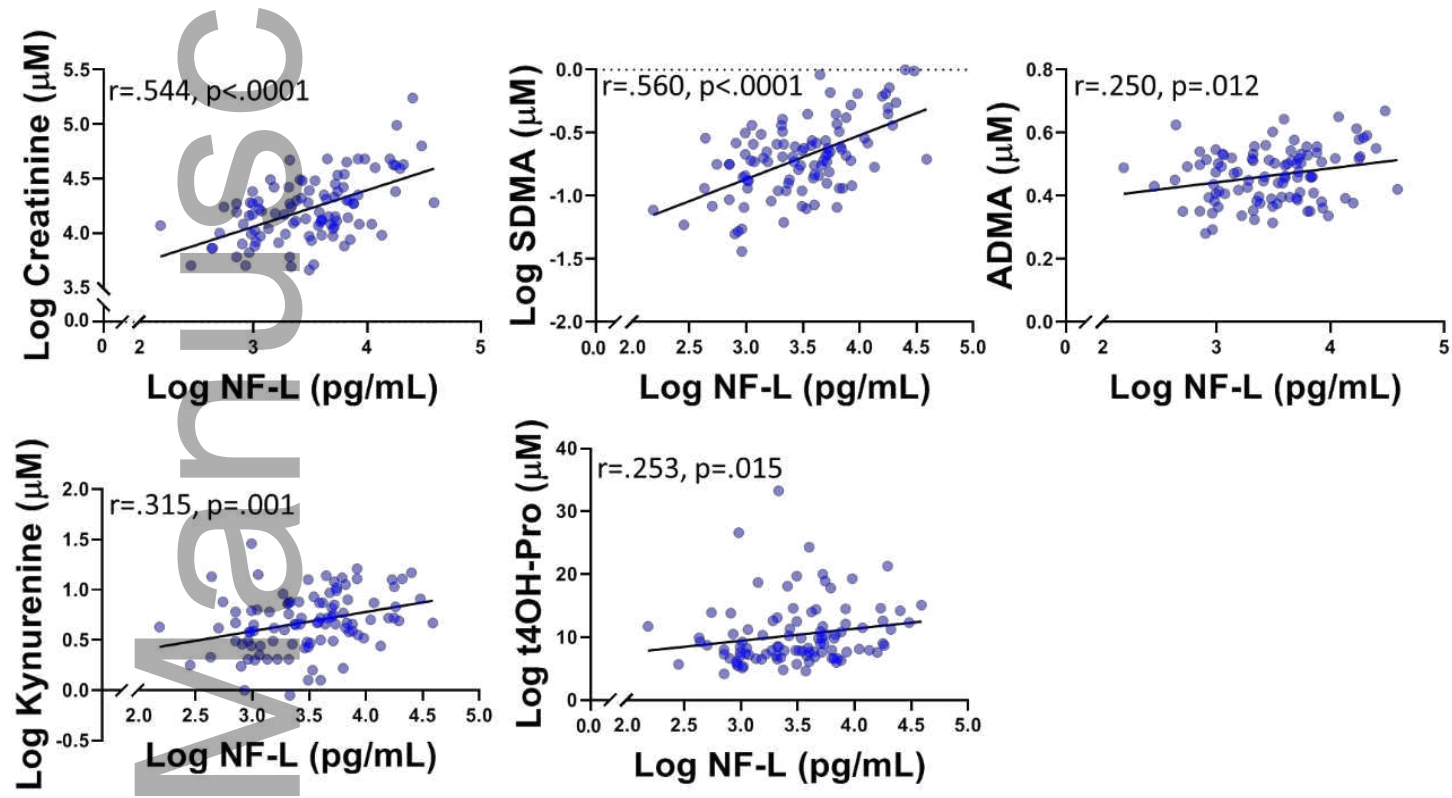

\section{B. Amino acids}
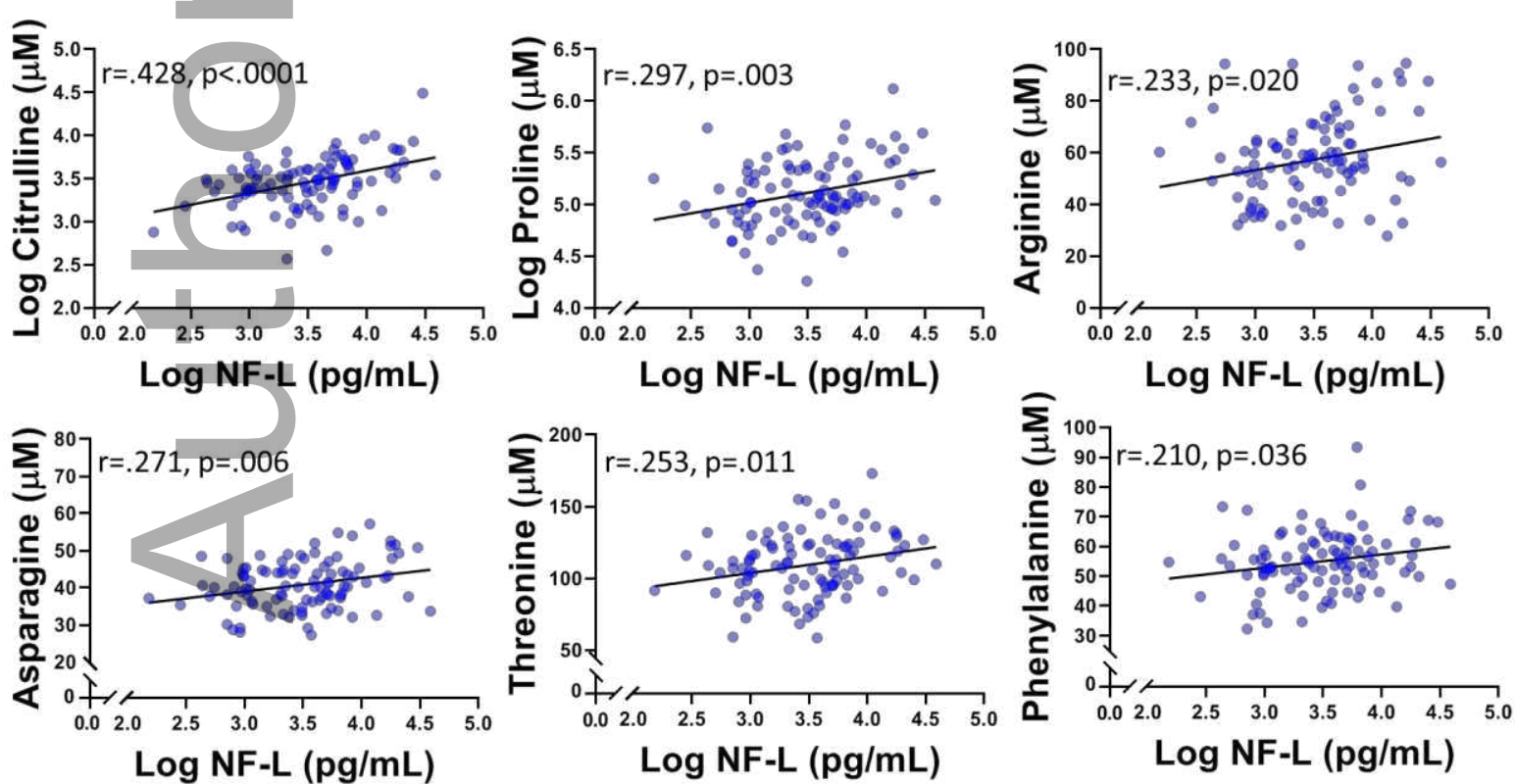

This article is protected by copyright. All rights reserved 


\section{Acylcarnitines}
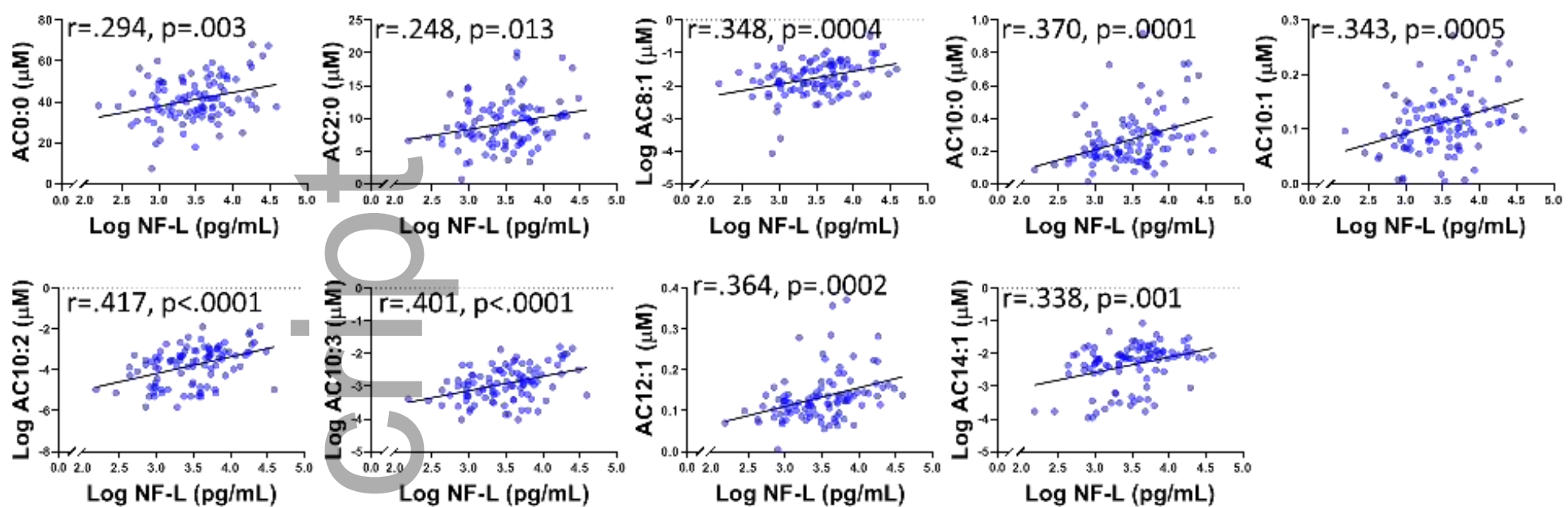

Log NF-L (pg/mL)

Log NF-L (pg/mL)

Log NF-L (pg/mL)
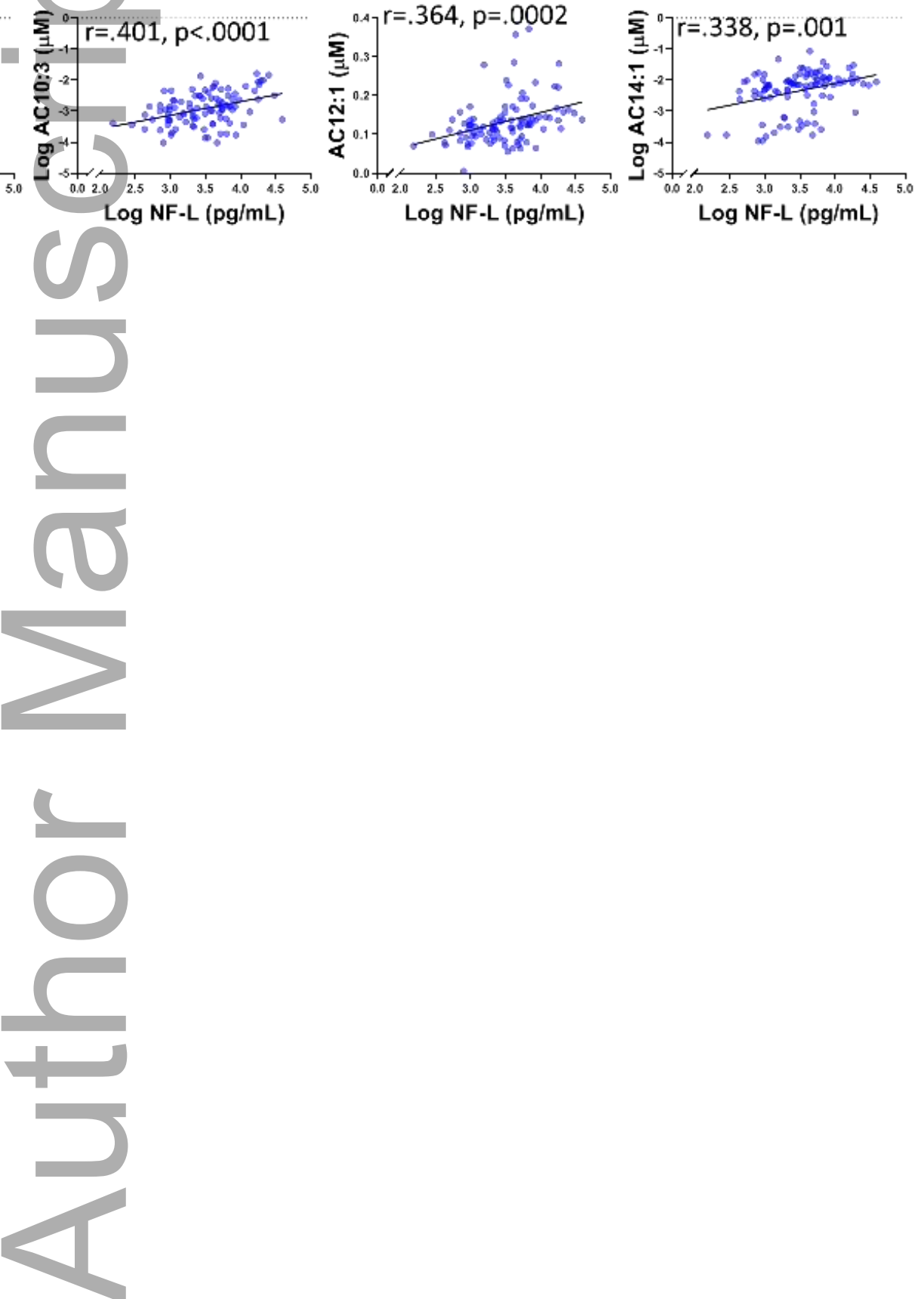

This article is protected by copyright. All rights reserved 


\section{University Library}

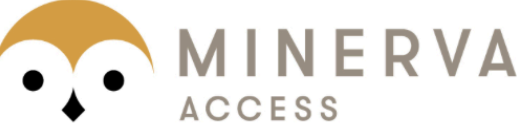

A gateway to Melbourne's research publications

Minerva Access is the Institutional Repository of The University of Melbourne

\section{Author/s:}

Chatterjee, P;Cheong, Y-J;Bhatnagar, A;Goozee, K;Wu, Y;McKay, M;Martins, IJ;Lim, WLF;Pedrini, S;Tegg, M;Villemagne, VL;Asih, PR;Dave, P;Shah, TM;Dias, CB;Fuller,

SJ;Hillebrandt, H;Gupta, S;Hone, E;Taddei, K;Zetterberg, H;Blennow, K;Sohrabi, HR;Martins, $\mathrm{RN}$

Title:

Plasma metabolites associated with biomarker evidence of neurodegeneration in cognitively normal older adults

Date:

2020-08-01

\section{Citation:}

Chatterjee, P., Cheong, Y. -J., Bhatnagar, A., Goozee, K., Wu, Y., McKay, M., Martins, I. J., Lim, W. L. F., Pedrini, S., Tegg, M., Villemagne, V. L., Asih, P. R., Dave, P., Shah, T. M., Dias, C. B., Fuller, S. J., Hillebrandt, H., Gupta, S., Hone, E. ,.. Martins, R. N. (2020). Plasma metabolites associated with biomarker evidence of neurodegeneration in cognitively normal older adults. JOURNAL OF NEUROCHEMISTRY, 159 (2), pp.389-402. https:// doi.org/10.1111/jnc. 15128.

Persistent Link:

http://hdl.handle.net/11343/276086 